\title{
La représentation de la musique et de la danse dans les œuvres de Post et Wagner : une archéologie des musiques noires au Brésil
}

The representation of music and dance in Post and Wagner's paintings: an archaeology of Brazilian Black music.

A representação da música e da dança nas pinturas de Post e Wagner: uma arqueologia da música afro-brasileira

Jean-Pierre Estival

\section{OpenEdition}

Journals

Édition électronique

URL : https://journals.openedition.org/jsa/13712

DOI : 10.4000/jsa. 13712

ISSN : 1957-7842

\section{Éditeur}

Société des américanistes

Édition imprimée

Date de publication : 17 septembre 2014

Pagination : $69-100$

ISSN : 0037-9174

Référence électronique

Jean-Pierre Estival, «La représentation de la musique et de la danse dans les œuvres de Post et Wagner : une archéologie des musiques noires au Brésil », Journal de la Société des américanistes [En ligne], 100-1 | 2014, mis en ligne le 01 janvier 2016, consulté le 03 septembre 2022. URL : http:// journals.openedition.org/jsa/13712 ; DOI : https://doi.org/10.4000/jsa.13712 


\title{
LA REPRÉSENTATION DE LA MUSIQUE ET DE LA DANSE DANS LES OEUVRES DE POST ET WAGNER : UNE ARCHÉOLOGIE DES MUSIQUES NOIRES AU BRÉSIL
}

\author{
Jean-Pierre ESTIVAL *
}

La période relativement brève de la colonisation hollandaise du Pernambouc (16301654) a été marquée par une entreprise documentaire sans précédent dans le Nouveau Monde, voulue par le comte Jean-Maurice de Nassau. Frans Janzsoon Post, chargé de peindre les paysages, les villages et leurs habitants, a très souvent représenté des tambourinaires noirs, accompagnés le plus souvent d'un petit groupe de danseurs. Dans ce corpus d'œuvres peintes au cœur du Siècle d'or hollandais, nous tentons de dégager un motif récurrent. Une gouache de l'aventurier Zacharias Wagner permet de compléter ces sources. À partir des analyses des historiens de l'art sur la place de la musique dans les œuvres néerlandaises contemporaines, et de l'étude de quelques rares sources écrites, nous dégageons les points principaux suivants : précision des descriptions organologiques, de la position des corps et nature de la représentation des performances. [Mots-clés: Brésil, ethnomusicologie historique, colonisation hollandaise, Frans Janzsoon Post, Zacharias Wagner, iconographie musicale.]

The representation of music and dance in Post and Wagner's paintings: an archaeology of Brazilian Black music. The relatively brief period of Dutch colonization in Pernambuco (1630-1654) corresponds to an exceptional documentary undertaking driven by JeanMaurice de Nassau. Frans Janzsoon Post, responsible for the painting of landscapes, villages and their inhabitants, often represented Black drummers accompanied by a few dancers. Here, we attempt to define a recurrent motif in the corpus of these works, belonging to the Dutch Golden Age. A gouache from the adventurer Zacharias Wagner completes the collection. By comparing art history analysis concerning the place of music in contemporary Dutch paintings with the few written sources, we emphasize the following points: precision regarding the organologic descriptions, the position of the musicians and the dancers' bodies, as well as the type of performance represented. [Key words: Brazil, historical ethnomusicology, Dutch colonization, Frans Janzsoon Post, Zacharias Wagner, musical iconography.]

\footnotetext{
* Centre de recherche en ethnomusicologie du LESC (UMR 7186, CNRS/Université Paris Ouest Nanterre la Défense), Nanterre [jphenryestival@yahoo.com].
}

Journal de la Société des Américanistes, 2014, 100-1, pp. 69-100. C Société des Américanistes. 
A representação da música e da dança nas pinturas de Post e Wagner: uma arqueologia da música afro-brasileira. O período relativamente curto da colonização holandesa em Pernambuco (1630-1654) foi marcado por atividades de documentação sem equivalente no Novo Mundo da epoca, sob o governo de Jean-Maurice de Nassau. O pintor Frans Janzsoon Post, encarregado de retratar as paisagens, as aldeias e seus moradores, representou muitas vezes percussionistas negros, geralmente acompanhados por dançantes. Uma aquarela do aventureiro Zacharias Wagner completa estas fontes. Neste corpus de obras pintadas no Século de Ouro holandês, tentamos revelar um motivo recorrente. A partir de análises de historiadores da arte sobre a importância da música nas obras holandesas contemporâneas, e do estudo de raras fontes escritas, esclarecemos os pontos seguintes: precisão das descrições organológicas e da posição dos corpos, e a maneira como a performance musical e de dança é representada. [Palavras chave: Brasil, etnomusicologia histórica, colonização holandesa, Frans Janzsoon Post, Zacharias Wagner, iconografia musical.]

Les musiques afro-américaines ont formé la matrice des musiques populaires depuis le début $\mathrm{du} \mathrm{xx}^{\mathrm{e}}$ siècle. Elles ont été à la base de développements particulièrement féconds dont le blues, le jazz, le samba, le tango, le reggae ou la salsa sont des exemples notoires. Issues du métissage entre formes d'origine européenne et formes d'origine africaine, lors d'un processus historique particulièrement cruel - la déportation, l'esclavage dans le monde de la plantation -, elles ont pris une place absolument considérable dans notre monde globalisé. On est frappé par leur diversité, mais aussi, et surtout peut-être, par leur extraordinaire créativité : peu d'éléments de modernité ou de mode dans le champ des musiques populaires sont exempts de référence aux formes afro-américaines. Le Brésil est l'un des pays où ce monde musical est le plus riche et le plus diversifié. Depuis les travaux pionniers de Carneiro (1982 [1961]) et d'Andrade (2002), l'apport des Noirs est aujourd'hui reconnu et valorisé (Sandroni 2001 ; Vianna 1999 [1995]) comme l'une des sources majeures de la musique brésilienne.

Nous voudrions présenter, dans cet article, une archéologie de ces formes, à partir d'un fonds documentaire exceptionnel qui date de la première moitié du $\mathrm{XVII}^{\mathrm{e}}$ siècle. Les tableaux de Frans Janzsoon Post et la gouache de Zacharias Wagner sont, du point de vue de l'iconographie musicale de la région, aussi souvent reproduits (par exemple, Tinhorão 2008 [1988], frontispice) qu'ils ont été peu étudiés de façon approfondie.

Après avoir rappelé le contexte de la colonisation hollandaise, nous introduirons la problématique de la représentation de la musique dans la peinture du Siècle d'or, puis nous évoquerons les quelques sources écrites sur le sujet. Les œuvres seront ensuite décrites, ce qui nous amènera à induire l'existence d'un motif type dans l'œuvre postienne. Nous tenterons enfin, sans sur-interpréter les sources, de comprendre comment ce motif a été intégré dans les compositions. 


\section{Le Brésil hollandais et le projet de Jean-Maurice de Nassau}

Dans l'histoire coloniale du pays, l'épisode hollandais ${ }^{1}$, relativement court (1630-1654), est certainement l'un des mieux connus : la documentation néerlandaise est immense, et elle a fait l'objet, depuis le milieu du XIX ${ }^{\mathrm{e}}$ siècle, de traductions et d'études importantes de la part d'historiens brésiliens et néerlandais. On citera en particulier Cabral de Mello (2010) - qui regroupe de nombreuses sources originales traduites ou transcrites en portugais moderne -, les œuvres classiques de Wäjten (1938 [1921]) et Boxer (2004 [1957]), mais surtout celle de Gonsalves de Mello (2001 [1947]) qui constitue une remarquable synthèse de l'histoire économique et sociale de la colonie. On trouvera une bibliographie à peu près complète des fonds manuscrits et imprimés chez cet auteur (ibid., pp. 281-286).

Le Brésil était une colonie portugaise depuis le début du $\mathrm{XvI}^{\mathrm{e}}$ siécle, et le Portugal était lui-même sous la domination de l'Espagne depuis 1580. Les Pays-Bas, devenus république des Provinces-Unies sortaient, au début du $\mathrm{XVII}^{\mathrm{e}}$ siècle, de leur terrible guerre d'indépendance contre l'Espagne ${ }^{2}$, et s'affirmaient déjà comme une puissance maritime de premier plan en Europe. La Geoctroyeerde Westindische Compagnie ou (G)WIC était une société commerciale - dotée d'une importante force militaire - qui avait le monopole du commerce avec l'Amérique et l'Afrique de l'Ouest. Conquis dès 1624, le Pernambouc devint la base de la conquête hollandaise du Nordeste brésilien. L'objectif de cette colonie était la production et la commercialisation du sucre, qui devait fournir des richesses considérables à la compagnie. Démanteler le Brésil portugais, et donc affaiblir indirectement l'Espagne, n'était sans doute pas non plus pour déplaire au gouvernement de la jeune république des Provinces-Unies.

En 1636, Jean-Maurice de Nassau ${ }^{3}$ est nommé gouverneur de la colonie. Il la marquera fortement de son empreinte jusqu'à son départ en 1644. Le Brésil hollandais ne lui survivra guère : après une phase de décadence et de défaites militaires successives face à l'insurrection portugaise, les Néerlandais quittèrent définitivement le Brésil en 1654. Chef de guerre et aussi homme de grande culture, Nassau arriva au Brésil avec des militaires, des scientifiques et des peintres comme Albert Eckhout (1610-1666) et Frans Post, assistés de Gillis Peeters (1612-1653), Caspar Schmalkalden (1616-1673) et Abraham Willaerts (16031669). Le projet documentaire naquit de la proximité que Jean-Maurice de Nassau entretenait avec les élites intellectuelles de son temps : prince européen, il souhaitait rapporter de son séjour les collections d'objets et la documentation scientifique les plus importantes possibles, afin de se voir reconnaître non seulement comme un grand militaire et un grand administrateur, mais aussi comme un interlocuteur des savants et de la haute noblesse cultivée d'Europe. L'amitié de Nassau avec Constantijn Huygens (1596-1687) ${ }^{4}$ permit ainsi le recrutement 
d'une équipe compétente. Les œuvres scientifiques de Georg Markgraf (16111644) et Willem Piso (1611-1678), de vivants récits (comme ceux du mercenaire strasbourgeois Ambrosius Richshoffer - 2004 [1677] - ou du pasteur Pierre Moreau - 1651), la compilation hagiographique de Barlaeus (1647) et, bien sûr, les œuvres peintes d'Eckhout (Brienen 2006), Post et Wagner forment un ensemble documentaire d'une densité tout à fait exceptionnelle pour le XVII ${ }^{\mathrm{e}}$ siècle colonial.

Nassau étendit considérablement la colonie par la force (du Sergipe au Maranhão), mais, surtout, se révéla être un administrateur hors pair, faisant preuve d'une grande tolérance religieuse ${ }^{5}$ tout en structurant la vie économique et sociale du Nordeste hollandais. Cette tolérance n'alla cependant pas jusqu'à supprimer ou adoucir l'esclavage des Noirs, qui constituait l'unique base économique de la production sucrière.

Le recueil d'une documentation précise visait à servir les intérêts politiques, administratifs et idéologiques de la conquête et de l'exploitation de la nouvelle colonie. Dans l'esprit même de Jean-Maurice de Nassau, les tableaux de Post et d'Eckhout participaient bien d'une entreprise documentaire. Lorsqu'il fit don à Louis XIV, à la fin de sa vie, de trente-quatre toiles de Post et de huit d'Eckhout ${ }^{6}$, il écrivit au roi : " [Je vous offre ces toiles] de manière à ce que Votre Majesté puisse voir ce beau pays, que rien n'égale au monde, reproduit en diverses œuvres qui représentent les hommes, les animaux, les poissons et les fruits, le tout en taille naturelle, [...] » (lettre de Clèves datée du 8 février 1679, in Cabral de Mello 2010, p. 324). Il dicta au peintre Paul de Mily, qui était chargé d'apporter les tableaux, un guide décrivant, œuvre par œuvre, son cadeau au roi. Ainsi, Jean-Maurice de Nassau fut à l'origine d'un projet encyclopédique qui préfigurait, en quelque sorte, l'aventure humaine et intellectuelle qui devait se développer plus d'un siècle plus tard, avec Diderot et d'Alembert, mais aussi Bougainville, Cook et Humboldt.

Pour les études afro-américaines et, en particulier, pour la musique, les peintures de Post et Wagner sont sans équivalent : au Brésil, il faudra attendre plus d'un siècle et quelques rares œuvres de Carlos Julião ( ca 1740-ca 1811), Jean-Baptiste Debret (1768-1848) ou Johan-Moritz Rugendas (1802-1858) pour avoir des représentations figurées de la musique et de la danse d'une certaine qualité. Rappelons enfin que les premiers enregistrements sonores de musiques noires au Brésil datent de 1937 (Oliveira Pinto 2004, p. 119).

Frans Janzsoon Post (1612-1680) arriva au Brésil avec la suite de JeanMaurice de Nassau, à l'âge de 24 ans. Né à Haarlem au sein d'une famille de peintres et d'architectes ${ }^{7}$, on sait peu de choses de sa vie et de sa formation (voir Corrêa do Lago et Corrêa do Lago 2007, pp. 23 et 80 ; Duparc 2012 ). Il vécut au Brésil entre 1637 et 1644 . Il y peignit dix-huit tableaux ${ }^{8}$ et fit d'innombrables dessins et croquis, pour la plupart aujourd'hui perdus. De retour à Haarlem, il y passa le reste de sa vie à peindre, presque exclusivement des thèmes brésiliens en 
utilisant certainement ses croquis. La qualité de ses tableaux déclina à partir du milieu des années 1650 tout comme, nous le verrons, leur aspect documentaire. Il sombra peu à peu dans l'alcoolisme et arrêta de peindre dix ans avant sa mort en 1680. Zacharias Wagner (1614-1668) a, quant à lui, peint une seule œuvre représentant des musiciens et danseurs. Nous évoquerons sa vie dans le paragraphe consacré à son Negertanz.

\section{Peindre la musique et Ses instruments}

Le Siècle d'or hollandais figure parmi les périodes les plus prestigieuses de l'histoire de l'art occidental (Alpers 1990). Les œuvres picturales étaient en effet extrêmement prisées par la bourgeoisie naissante, qui commençait à s'enrichir fortement du fait du commerce et des premières conquêtes coloniales, alors même que la guerre de Quatre-Vingts Ans était encore latente ${ }^{9}$. Dans toutes les villes, il y avait de nombreux artistes, souvent organisés en guildes, qui se disputaient âprement un marché à la fois très concurrentiel et très cadré dans ses canons esthétiques (Bergström 1956 ; Zumthor 1960). Le XVII ${ }^{\mathrm{e}}$ siècle hollandais a été riche en représentations de scènes musicales ou d'instruments de musique (entre 10 et $12 \%$ de l'ensemble de la production du siècle ${ }^{10}$ ). De très grands maîtres, comme de nombreux peintres de moindre importance, y consacrèrent une partie de leur œuvre (Fischer 1972). La religion protestante, majoritaire, permettait aux peintres de se consacrer à d'autres thèmes que les sujets religieux, historiques ou hagiographiques qui s'imposaient aux artistes vivant dans la péninsule ibérique catholique. C'est certainement l'une des raisons pour lesquelles le projet brésilien de Nassau a pu voir le jour, et aussi pourquoi les formes musicales et chorégraphiques afro-brésiliennes, considérées par l'Église catholique (ici hispanoportugaise jusqu'en 1640) comme des manifestations diaboliques, ont pu être représentées par Post et Wagner.

$\mathrm{Au} \mathrm{XIX}^{\mathrm{e}}$ siècle, la critique n'hésitait pas à comparer les scènes de genre de l'âge d'or hollandais à la naissante photographie (Thoré, cité in Kyrova 1994, p. 36). L'histoire de l'art contemporaine s'intéressant aux représentations de la musique dans la peinture hollandaise s'est considérablement éloignée de cette vision naïve, montrant que les tableaux relèvent de la construction d'un univers symbolique riche et complexe (Leppert 1977, 1993 ; Kyrova 1994). Dans les Pays-Bas du XVII ${ }^{\mathrm{e}}$ siècle, on assiste ainsi à la distinction entre musiques sonores et bruyantes (les célèbres œuvres de Steen, Brueghel, Teniers le Jeune, Metsu ou Molenaer) et la musique d'art évoquée dans les tableaux de Vermeer, van Kessel, van Schoor ou van Tilborgh (Mirimonde 1961, 1962). Cette distinction renvoie à l'opposition art public/art privé, ce dernier étant l'apanage de la bourgeoisie

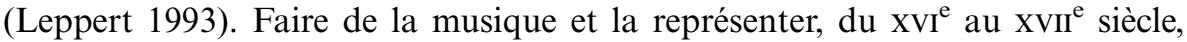
répondait tout d'abord aux codes exprimés par deux groupes : les religions 
hégémoniques et les classes sociales hégémoniques; double et immense distance donc, géographique et sociale, par rapport aux danses et tambours des esclaves brésiliens. Cela montre l'originalité du travail de Post, mandaté par Jean-Maurice de Nassau, et le caractère exceptionnel de son œuvre dans l'histoire de la représentation de la musique dans l'art pictural. Les artistes du Siècle d'or, comme l'a montré Leppert (1975), ont produit généralement des représentations de musiciens populaires conformes aux attentes et aux habitus de leurs acheteurs. Post évoluait, aux Pays-Bas, dans un environnement où la plupart des artistes étaient des spécialistes : il occupait, avec Eckhout, la « niche brésilienne ", s'assurant ainsi une position hégémonique sur une part très spécifique du marché de l'art. Étudiant le style et la technique de Post, Gordon a souligné l'originalité des œuvres:

Much has been written about their extraordinary naturalism and lack of obvious artistic debt to anything that precedes them in Dutch art. This can only partly be explained if one takes the view that they would not have been considered by the artist and his peers in Brazil as paintings in the conventional sense, but as a record in paint of what he and they saw: part of the process of registering and recording the unfamiliar natural world that surrounded them, as Posts fellow scientists were doing in other ways. (Gordon 2007, p. 67)

Les instruments afro-brésiliens représentés par Post et Wagner (tambours, hochets, racleurs) ne faisaient pas partie de l'instrumentarium habituellement peint par les artistes néerlandais. Le luth ou le virginal (Kyrova 1994, pp. 44-47) étaient chargés, dans les nombreuses représentations dont ils firent l'objet, de références symboliques complexes et parfois contradictoires (de l'expression de la fidélité maritale à celle des affres du désir). Ces références étaient attendues et comprises par les commanditaires des œuvres. Rien de tel pour les percussions des esclaves, bien évidemment. Il est certain que, pour un Néerlandais du XVII ${ }^{\mathrm{e}}$ siècle achetant ou regardant un tableau de Post, la symbolique proprement musicale ou chorégraphique relevant des chants et danses des Noirs était quasi inexistante. Les « anciens du Brésil » revenus au pays trouvaient des éléments porteurs de mémoire dans la représentation des corps et des sons, mais il est plus que douteux que ces personnes aient pu avoir accès, lors de leur séjour brésilien, aux riches éléments culturels et cultuels liés aux pratiques musicales et chorégraphiques de leurs esclaves. En tout cas, nous n'en avons pas trace dans les chroniques.

Avant d'aborder les œuvres et leur interprétation, on ne peut oublier que Jean-Maurice de Nassau conçut son projet documentaire en lien étroit avec Constantijn Huygens, lui-même compositeur et musicien impliqué dans la vie musicale de son temps ${ }^{11}$. Il est cependant très peu probable qu'une consigne ait pu être donnée quant à la représentation de formes musicales et chorégraphiques afro-américaines. Celles-ci n'étaient, en effet, sans doute pas considérées comme de la musique ou de la danse, mais bien plus vraisemblablement, nous le verrons, comme des éléments de pratiques exotiques intégrables dans un tableau 
parallèlement, en quelque sorte, aux musiques populaires qui étaient perçues comme des éléments de la vie ordinaire, et non comme de l'art.

Nous terminerons en remarquant qu'aucun des historiens de l'art s'intéressant à la musique n'a, à notre connaissance, analysé la présence de musiciens noirs dans les œuvres de Post ou de Wagner, bien que le premier jouisse d'une notoriété certaine. De leur côté, les expositions thématiques et leurs catalogues spécialisés (Moens et Kochelbergh 1994 ; Buijsen et Grijp 1994) ne font pas référence à ces œuvres ${ }^{12}$.

\section{LES SOURCES ÉCRITES CONCERNANT LES MUSIQUES NOIRES DU BRÉSIL AU XVII SIÈCLE}

Tenter d'analyser les représentations de la musique dans les œuvres du $\mathrm{XVII}^{\mathrm{e}}$ siècle suppose que l'on s'intéresse aux descriptions écrites, littéraires ou factuelles, de ces formes. Les fonds écrits sur les musiques populaires du XVII ${ }^{\mathrm{e}}$ siècle sont très peu nombreux, au Brésil comme ailleurs. Rappelons, à titre comparatif, que les musiques populaires européennes ne seront décrites avec une certaine précision qu'à partir de la seconde moitié du XIX ${ }^{\mathrm{e}}$ siècle (Lesure 1954 ; Charles-Dominique 1994 ; Gétreau 1997) ; les références écrites antérieures sont principalement des actes juridiques (concernant les contrats de musiciens, les successions ou la corporation ménétrière) ou administratifs (ordre public, etc.). En Amérique, les esclaves ne pouvaient prétendre ni à des corporations ménétrières ni, bien évidemment, à des actes successoraux. Ce sont donc des récits de voyageurs ou des chroniques locales qui nous transmettent de bien maigres éléments, comme à la même époque aux Antilles (Labat 1993 [1698], pp. 230231). Seul le tableau de Wagner joint une description écrite au dos de l'œuvre picturale ( $c f$. infra). Cette pénurie de mentions écrites renforce l'intérêt que l'on doit porter aux œuvres peintes, qui constituent donc la source principale pour la documentation du sujet qui nous occupe. Nous n'avons pas eu la possibilité, ni les compétences épigraphiques et linguistiques, d'aborder les sources coloniales néerlandaises. Cela mériterait une recherche approfondie qui dépasse la cadre de cet article. Plusieurs des chroniqueurs ayant relaté les activités musicales des esclaves brésiliens au XVII ${ }^{\mathrm{e}}$ siècle étaient des Français, qui, malheureusement, n'étaient pas exactement contemporains de l'aventure hollandaise. On peut néanmoins raisonnablement considérer que les formes qu'ils ont évoquées ou décrites n'étaient pas fondamentalement différentes de celles pratiquées entre 1630 et 1654 .

La première mention de la musique jouée par des esclaves brésiliens au $\mathrm{XVII}^{\mathrm{e}}$ siècle provient du récit de François Pyrard de Laval, relation d'un voyage qui l'amena jusqu'aux Moluques. Lors de son escale au Brésil en 1610, dans la région de Bahia, il rencontra un riche seigneur portugais ayant un domestique français, 
né près de Marseille : « Ce François qui demeuroit avec luy estoit Musicien, \& ioüeur d'instruments, $\&$ ce Seigneur l'avoit pris pour apprendre vingt ou trente esclaves, qui tous ensemble faisoient un concert de voix \& d'instruments dont ils ioüoyent à toute heure. » $(1619$, p. 333.) On remarquera que les Noirs, et c'est une constante dans l'histoire afro-américaine, jouent ici certainement la musique de leurs maîtres, avec des instruments européens. Pyrard complète : « C'est un grãd plaisir tous les festes \& dimãches, de voir assembler les esclaves, hommes \& femmes, qui dansent, \& jouënt en public és places \& ruës ; car ces jours là ils ne sont sujets à leurs maistres. » (Ibid., p. 339.)

Parmi les voyageurs, on citera aussi la courte note d'Urbain Souchu de Rennefort, souvent reprise dans les ouvrages brésiliens. En voici un extrait d'une édition française :

Ils ne laissent pas dans cette dure captivé [captivité] de se réjoüir quelquefois. Le Dimanche dixième Septembre 1666, ils firent leur feste à Fernambouc ${ }^{13}$. Aprés avoir esté à la Messe au nombre environ de quatre cens hommes \& de cent femmes, ils éleurent un Roy \& une Reyne, \& marcherent par les ruës chantans, dansans, \& récitans des vers qu'ils avoient faits, precedez de hautbois, de trompettes et de tambours de basque. Ils estoient vestus des habits de leurs Maîtres \& Maîtresses, avec des chaisnes d'or \& des pendants d'oreilles d'or \& de perles ; quelques-uns masquez. (1701 [1688], p. 292-293)

En ce qui concerne les (très rares) sources secondaires portugaises, les deux ouvrages de Tinhorão (2000, 2008 [1988]) sont les références de synthèse les plus accessibles. On notera la présence de Noirs dans les processions catholiques du Corpus Christi ${ }^{14}$ et la mention de danses nocturnes bruyantes et lascives ${ }^{15}$.

Alors que Post, on le verra, a toujours peint des scènes musicales dans une ambiance calme et paisible de vie quotidienne, il est assez piquant de noter que la seule mention portugaise de pratique musicale pendant la colonisation néerlandaise se réfère à l'acclamation du chef de la révolte contre les Hollandais, João Fernandes Vieira : en 1645, les guerrilheiros pernamboucains « [...] acclamèrent par trois fois la victoire, et la célébrèrent au son des chalemies, caisses et trompettes ${ }^{16}$, ce qui firent aussi nos Noirs Mina, jouant leurs trompes, flûtes et tambours ${ }^{17} \gg$ (Frei Manuel Calado, cité in Tinhorão 2008 [1988], p. 36).

Le poète Gregório de Matos ${ }^{18}$ évoqua, dans la seconde moitié du XVII ${ }^{\mathrm{e}}$ siècle, les calundus ${ }^{19}$, terme pouvant signifier à la fois les dieux africains et, par métonymie, les danses et cérémonies afro-brésiliennes qui s'y réfèrent. Ces danses attiraient non seulement les esclaves, mais aussi les Européens qui venaient s'y distraire et chercher des femmes.

Les chroniqueurs n'ont pas décrit de façon claire des formes musicales où seuls les tambours, le chant ou la danse étaient utilisés : peut-être ont-ils effectué des séjours trop courts, et certainement très surveillés par les autorités, pour avoir le loisir de témoigner de formes plus quotidiennes telles que celles représentées par Post. Nous y reviendrons. 


\section{Le corpus : musiciens et danseurs dans l'euvre de Post}

Dans les pages suivantes, l'indication « $\mathrm{CR}\{\mathrm{n}\}$ » (« $\mathrm{n}$ » étant le numéro de l'œuvre) renvoie au catalogue raisonné de Corrêa do Lago et Corrêa do Lago (2007). Ce dernier comprend les 155 tableaux confirmés de Post ; y sont joints 11 tableaux d'attribution douteuse, 40 œuvres rejetées et classées par ordre chronologique, les 57 dessins qui sont parvenus jusqu'à nous ${ }^{20}$ ainsi qu'une trentaine de gravures. Nous présenterons quelques œuvres où figurent des musiciens et danseurs, avant de relever des récurrences sur l'ensemble du corpus.

L'identification des musiciens, essentiellement des tambourinaires, est assez simple et peut être précise dans les œuvres. Il n'en va pas de même pour les danseurs(euses). La qualification même de la « danse » relève d'une problématique choréologique qui dépasse de loin le cadre de cet essai. Si certaines représentations sont explicites (par exemple CR $\{24\},\{25\},\{42\}$, etc.), il est parfois assez spéculatif de considérer que certains personnages dansent et d'autres pas. Le peintre représente souvent un motif avec des personnages noirs, l'un des bras tendu ou écarté du corps, sans la présence de musiciens, et donc figurant sans doute une conversation animée ${ }^{21}$... et parfois le même motif est fort proche de tambourinaires en situation de jeu. Il est donc assez difficile de décider parfois si ces personnes sont représentées en mouvement de danse. Nous éviterons donc soigneusement de faire une analyse quantitative sur ce sujet dans le corpus, tout en indiquant, avec une marge d'erreur forte, leur nombre tel qu'il nous est apparu. De plus, l'observation contemporaine des formes chorégraphiques afro-brésiliennes de la région (ou de l'aire caraïbe) montre un continuum certain entre le mouvement dansé, acte corporel et social délibéré et organisé, et le simple fait de « bouger » au son de la musique, en discutant, buvant, etc.

Nous ferons la même remarque en ce qui concerne le chant. Si nous savons, tant en Afrique que dans les Amériques noires, que les tambours accompagnent très souvent la voix humaine, il est extrêmement difficile d'observer que, dans les œuvres, tel personnage chante et un autre pas.

Des 7 ans de son séjour au Brésil, nous ne conservons que 7 tableaux de Post, sur au moins 18 réalisés dans le pays. Aucun d'entre eux ne représente des musiciens ou des danseurs. Dans la suite de son œuvre, peinte en Hollande d'après ses croquis brésiliens, on trouve 25 représentations de danseurs et musiciens. C'est une proportion importante sur l'ensemble des 155 tableaux : il est difficile de prétendre qu'il s'agit là de vraies statistiques (en raison d'œuvres perdues ou non encore identifiées), mais environ $16 \%$ des œuvres qui nous sont parvenues contiennent des sujets musicaux. Ce chiffre est un peu plus important que la moyenne des représentations de thèmes musicaux dans les peintures du Siècle d'or (on l'a vu, moins de $12 \%$ ). 
Les peintures de Frans Post ont été classées en phases successives par Corrêa do Lago et Corrêa do Lago (2007), selon des critères stylistiques et, aussi, selon l'évolution du marché des acheteurs ou commanditaires du travail de Post.

\section{La deuxième phase (1645-1660)}

Cette partie de l'œuvre hollandaise de Post (40 peintures) est, avec la première phase, brésilienne, qui malheureusement ne présente pas de musicien ou de danseur, la plus précise et la plus crédible sur le plan documentaire, pour plusieurs raisons : d'une part, Post (alors âgé de 33 à 48 ans) était en pleine possession de ses moyens artistiques, d'autre part, sa mémoire visuelle et vécue du Brésil, celle de ses lumières et de ses ambiances était certainement encore vive; enfin, la plupart des acheteurs de ses toiles étaient eux-mêmes des " anciens du Brésil hollandais », attachés à la remémoration par la peinture de leurs expériences et de leurs sensations, nous y reviendrons. Pour les satisfaire, le peintre leur donnait des détails précis et respectait l'aspect globalement plausible des choses dans ses compositions. Cet aspect documentaire encore vivant peut être confirmé par le fait que l'artiste ne se recopie pas, et ne mélange pas les compositions, contrairement à ce qui se passe dans les deux phases suivantes. Bien qu'il soit, dans sa conception des paysages, moins original que dans sa première phase brésilienne, Gordon a noté : "His working practice, however, remained unlike that the most of his Dutch contemporary landscapists, with the oft-noted exception of Cornelis Vroom. » (2007, p. 73.)

Il se gardait pourtant, on y reviendra, de représenter les aspects les plus désagréables ou les plus pénibles de la vie réelle de la colonie. Images d'un Brésil exotique et idéal, où les compositions du peintre sélectionnent soigneusement la représentation des souvenirs agréables des clients et anciens Néerlandais brésiliens ? C'est une thèse abondamment développée par Oliveira (2005a, 2005b). Cet auteur a ainsi pu parler de l'« invention d'un nouveau monde ». Sans doute, et l'on notera alors que la musique et la danse des Noirs en fait partie. 


\section{Un village au Brésil (Figures 1 et III dans le cahier en couleurs)}

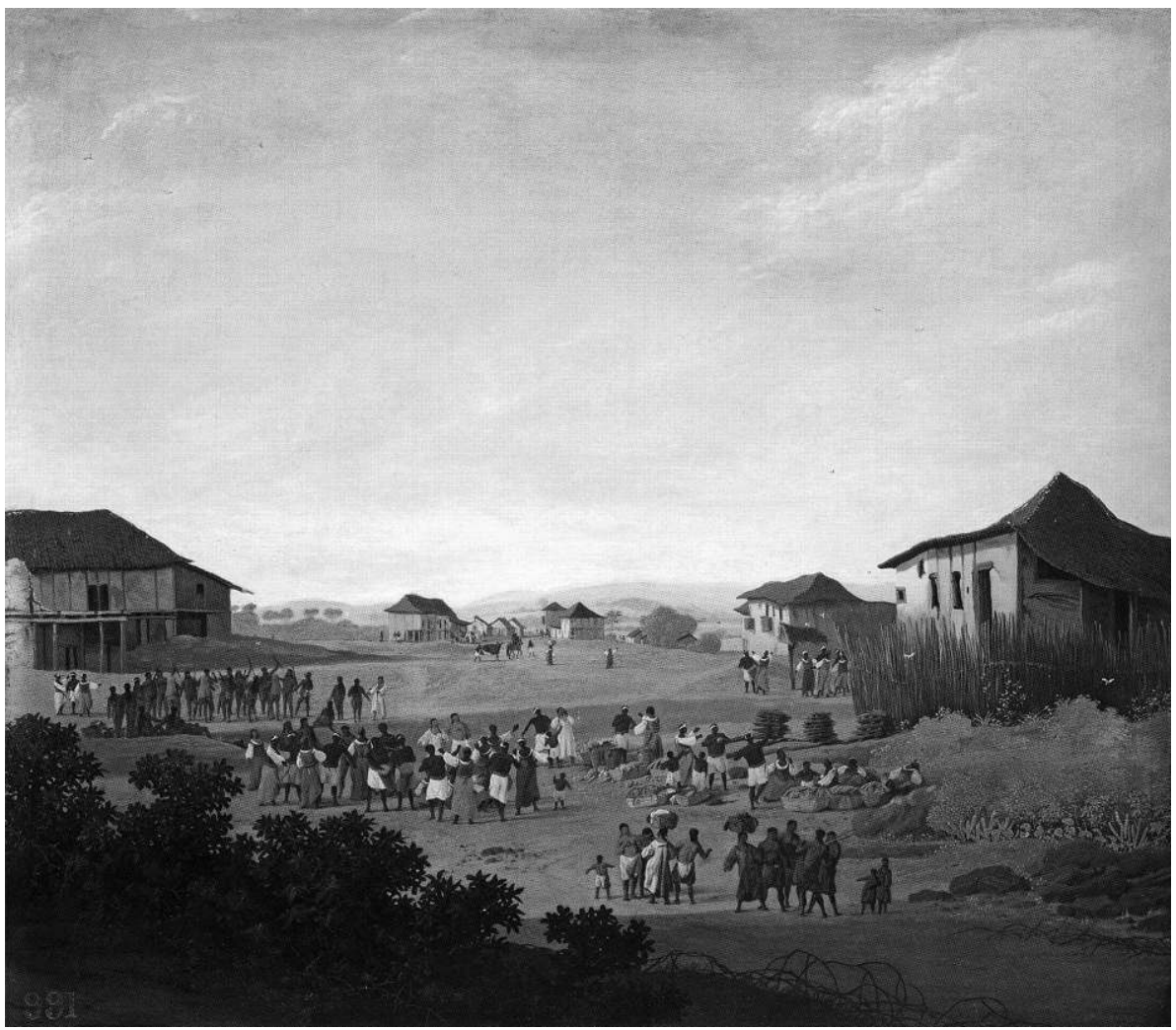

FIG. 1 et III dans le cahier en couleurs - CR \{18\} Frans J. Post. Un village au Brésil, 1652. Huile sur toile. 51,1 x 59,1 cm. The Royal collection, Windsor Castle, Windsor. « Royal Collection Trust / (C) Her Majesty Queen Elizabeth II $2014 »$.

Cette œuvre remarquable, datée de 1652, montre trois groupes de personnages dansant ou, à tout le moins, gesticulant :

- dans le fond à gauche, des Amérindiens, reconnaissables à leur absence de vêtements et à leurs armes brandies (arcs et flèches, massues). C'est la seule représentation d'un groupe conséquent d'Amérindiens (vraisemblablement des Tupis de la côte) en situation de mouvement ;

- au centre du tableau, un groupe d'une quinzaine de personnes, dont au moins trois ou quatre Amérindiens, dansent (ou assistent de près à une danse) menée instrumentalement par deux tambours. Un groupe de danseurs est identifiable : au premier plan, en rond, levant les bras (six personnes). Derrière les deux 
instrumentistes jouant côte à côte, un groupe formé de quatre personnes, esquissent peut-être des mouvements chorégraphiques, bien que cela ne soit pas très clair;

- en haut sur la droite, sous un auvent, un groupe de cinq Noirs dansent au son d'un tambour (dont deux couples face à face), accompagnés par un tambourinaire seul, devant lequel danse également une femme ;

- enfin, dans le fond, un cavalier européen ${ }^{22}$ suivi de deux esclaves portant peut-être un palanquin.

Tous les éléments qui nous intéressent, et qui seront reproduits dans les œuvres suivantes, sont déjà présents ici : groupe restreint de danseurs, un ou deux instrumentistes.

\section{Paysage de varzea avec hutte (Figures 2 et IV haut dans le cahier en couleurs)}

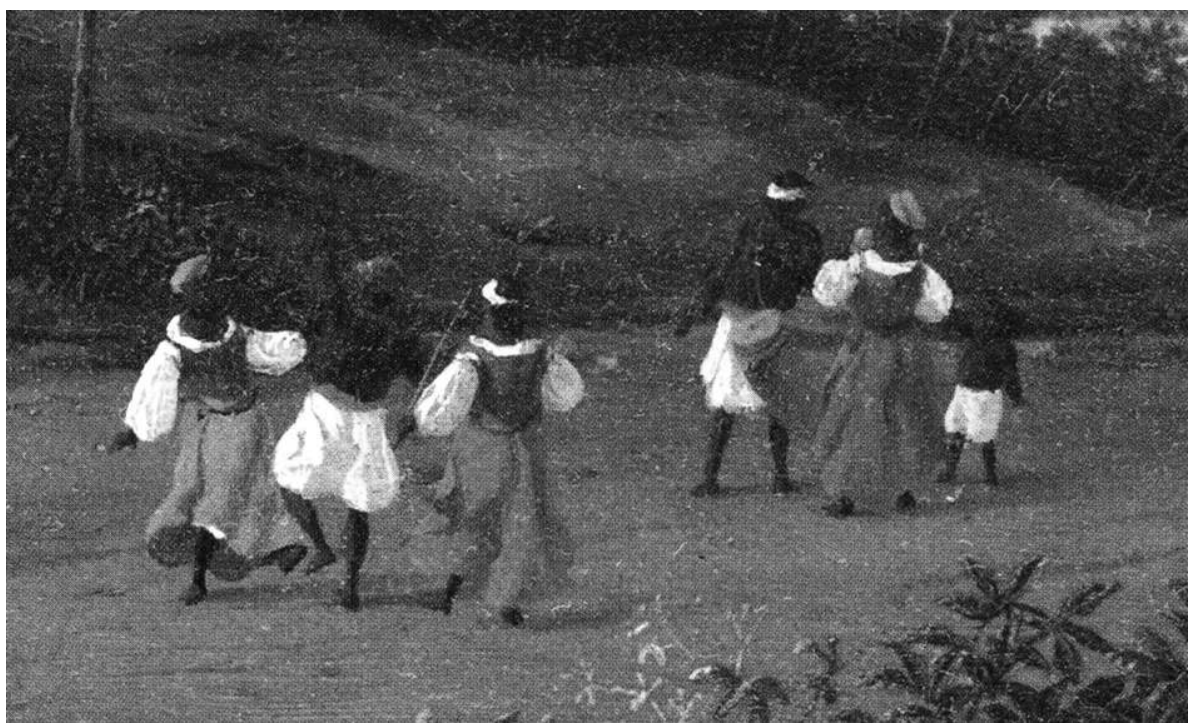

Fig. 2 et IV haut dans le cahier en couleurs - Détail de CR \{39\} Frans J. Post. Paysage de varzea avec hutte, 1658. Huile sur toile. 40,5 x 54,5 cm. Ella Gallup Summer and Mary Catlin collection, Wadsworth Atheneum, Hartford.

Daté de 1658, ce tableau montre, au centre et un peu éloignés du groupe principal d'esclaves placé devant les maisons, trois Noirs dansant, avec tambourinaire. Une des femmes, de dos, tient à la main un objet rond, de petite taille, qu'elle semble frapper avec une longue et fine baguette. Cela pourrait être une 
calebasse frappée, instrument non standard ${ }^{23}$, peut-être improvisé sur place, ou alors s'agit-il d'une recomposition erronée, de mémoire, de l'artiste, qui peignait à cette époque en Hollande, d'après ses croquis ${ }^{24}$. Autre hypothèse : il pourrait s'agir d'une cloche, mais la forme générale ne correspond pas.

\section{Maison en construction à Serinhaem (Figure 3 et IV bas dans le cahier en couleurs)}

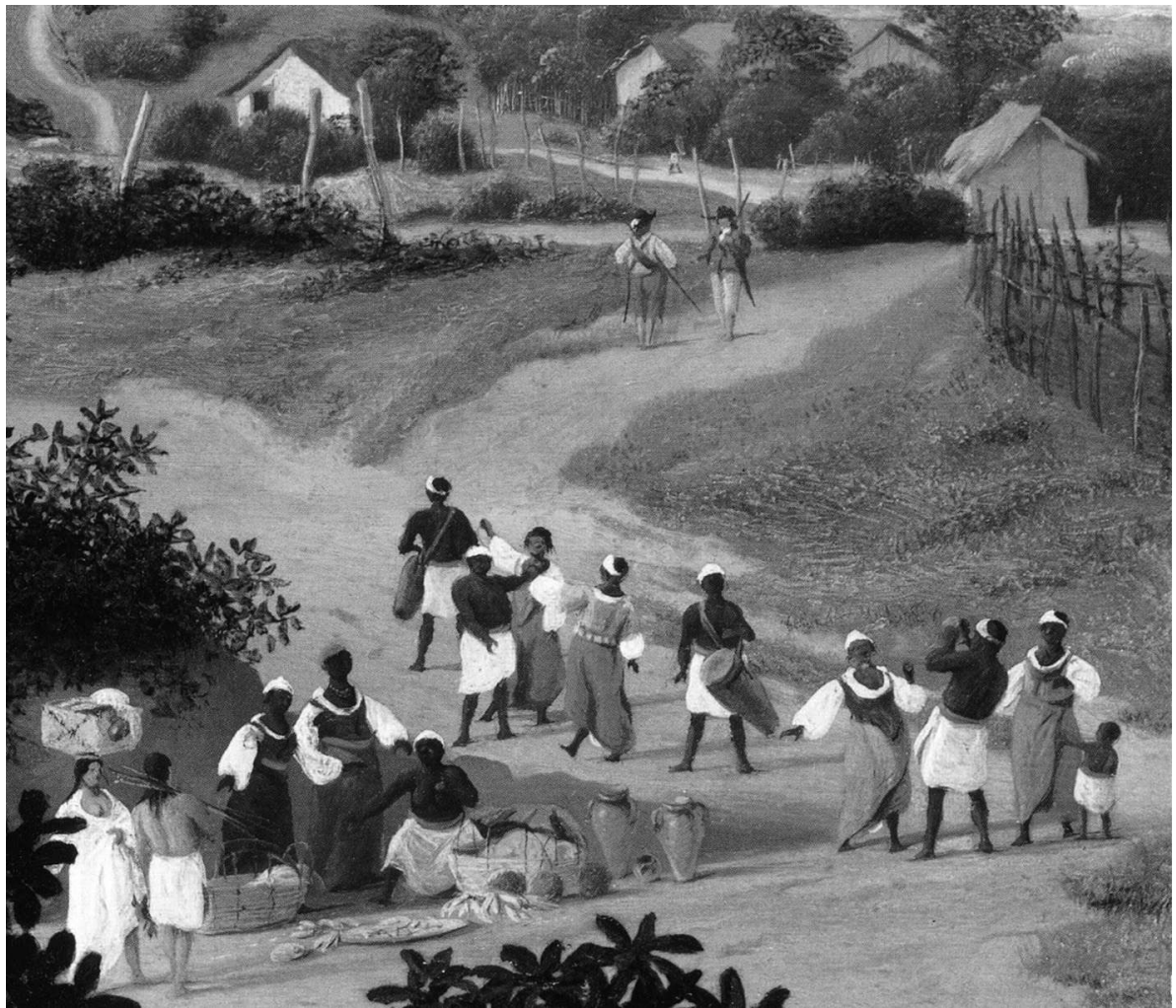

FIG. 3 et IV bas dans le cahier en couleurs - Détail de CR \{42\} Frans J. Post. Maison en construction à Serinhaem, ca 1655-1660. Panel, $46 \times 70 \mathrm{~cm}$, inv. 1127. Don de Willem baron van Dedem aux «Friends of the Mauritshuis Foundation », 2002.

Cette œuvre a été remarquée par Corrêa do Lago et Corrêa do Lago comme l'une des plus exemplaires de l'œuvre de Post ${ }^{25}$, et en particulier de sa seconde phase (1645-1660). On peut lire : "The often-depicted scene of slaves dancing to the sounds of an atabaque drum in the foreground is renderred convincingly, with utmost care in the execution of the figures, including the drum player at the center. » 
(2007, p. 180). En fait, deux tambourinaires et un joueur de hochet accompagnent quatre danseuses et un danseur, à un croisement de routes. Deux Européens sont à quelques mètres derrière, des Amérindiens aussi sont présents, partiellement cachés par le repoussoir végétal au premier plan du tableau.

\section{Le couvent franciscain d'Iguaraçu (Figures 4 et $\mathrm{V}$ dans le cahier en couleurs)}

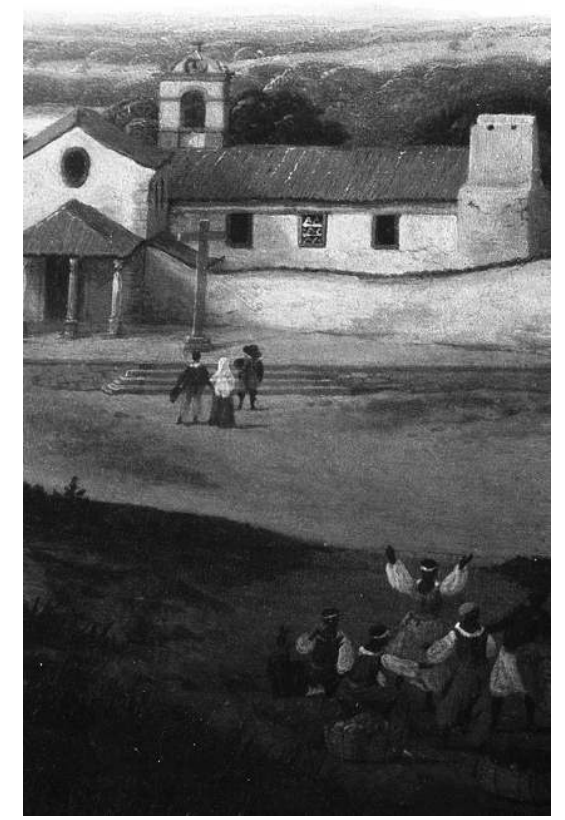

FIG. 4 et V dans le cahier en couleurs - Détail de CR $\{44\}$ Frans J. Post. Le couvent franciscain d'Iguaraçu, 1659. Huile sur toile. 50 x $41 \mathrm{~cm}$. Collection privée, São Paulo.

La petite ville d'Iguarassu (nouvelle orthographe) a sans doute été souvent visitée par Post, comme l'attestent différents tableaux qu'il en fit. Située à $35 \mathrm{~km}$ au nord de Recife, c'est l'un des premiers lieux colonisés du Nordeste, et le siège de l'une des plus anciennes églises du pays. Aujourd'hui, c'est l'un des hauts lieux de la culture populaire pernamboucaine, en particulier du fait de son maracatu ${ }^{26}$, Estrela brilhante, dont la famille responsable pratique aussi le coco. Le couvent et la croix (dont une reproduction est toujours en place ${ }^{27}$ ) sont exposés au soleil, alors qu'un groupe de cinq Noirs est regroupé en bas à droite du tableau, dans 
l'ombre : l'un joue du tambour, deux femmes dansent et deux autres sont assises, l'une tendant les bras vers une danseuse.

\section{Les troisième (1661-1669) et quatrième (1670-1680) phases}

La troisième phase a été la plus brillante du point de vue de la technique et de la qualité des compositions. C'est aussi la plus prolifique (69 œuvres) et la plus libre, sans doute parce que les clients de l'époque, qui n'avaient pas nécessairement visité le Brésil, exigeaient des compositions exotiques et brillantes :

Les nouveaux et prospères clients de Post voulaient désormais des compositions riches en détails tropicaux, arbres, moulins à sucre, végétation exotique, [...], et surtout Indiens et nombreux esclaves noirs (de préférence en train de danser). Ainsi, durant les années 1660, Post choisit, pour répondre aux commandes de ses clients, des arrangements décoratifs de ces divers éléments « brésiliens » dans des tableaux qui dès lors ne gardent qu'un très faible rapport avec la réalité qu'il avait observée, mais dans lesquels aucun élément n'était en soi inexact ou inventé. (Corrêa do Lago et Ducos 2005, p. 24.)

L'artiste a ainsi mêlé des éléments de la vie brésilienne, cela avec un moindre souci d'authenticité que lors de la phase précédente.

Peintes pendant les dix dernières années de sa vie (entre 58 et 67 ans), les 39 œuvres de la quatrième phase montrent une vue et une technique déclinantes, dues sans doute en partie à l'alcoolisme du peintre (Corrêa do Lago et Corrêa do Lago 2007, p. 297).

\section{La définition d'un motif récurrent}

L'analyse de ces œuvres a permis de dresser le tableau suivant qui enregistre le nombre d'instrumentistes (par groupe cohérent dans un tableau, le tambour étant l'instrument par défaut) et de danseurs(euses) représentés dans chaque œuvre (Figure 5).

Les scènes musicales peintes par Post comprennent donc un motif type, qui peut être juxtaposé, dans la composition du tableau, à d'autres motifs, comme par exemple des marchandes assises ou des repoussoirs. Caractérisons-le : un (plus rarement deux) instrumentiste, jouant avec les mains d'un tambour unimembranophone conique, accompagnant quelques (environ trois ou quatre) danseurs(euses) (généralement plus de femmes que d'hommes). Nous appellerons ce groupe « motif principal ». Nous montrerons plus loin que Post a intégré dans ses paysages ce motif principal, à la fois comme marqueur d'une construction de l'image du Brésil, et comme élément documentaire observé avec précision, puis rapporté parmi d'autres personnages meublant ses tableaux. 


\begin{tabular}{|c|c|c|c|}
\hline 1 instrumentiste & 2 instrumentistes & danseuses & danseurs \\
\hline $\mathrm{CR}\{18\}$ & $\mathrm{CR}\{18\}$ & $8(4+4)$ & $4(2+2)$ \\
\hline CR $\{20\}$ (cloche/hochet ?) & & 1 & 2 \\
\hline $\mathrm{CR}\{24\}$ & CR $\{24\}$ (tambour + cloche/hochet ?) & $9(3+6)$ & $5(2+3)$ \\
\hline $\mathrm{CR}\{25\}$ & & 3 & 3 \\
\hline $\mathrm{CR}\{26\}$ & & 1 & 1 \\
\hline $\mathrm{CR}\{30\}$ & & 3 & 3 \\
\hline \multirow[t]{2}{*}{$\mathrm{CR}\{38\}$} & & 3 & 1 \\
\hline & CR $\{39\}$ (tambour + cloche/hochet ?) & 2 & 1 \\
\hline $\mathrm{CR}\{42\}$ & CR $\{42\}$ (tambour + hochet) & 3 & 1 \\
\hline $\mathrm{CR}\{44\}$ & & 2 & \\
\hline $\mathrm{CR}\{45\}$ & & 2 & 1 \\
\hline $\mathrm{CR}\{65\}$ & & 2 & 2 \\
\hline $\mathrm{CR}\{70\}$ & & 2 & 1 \\
\hline $\mathrm{CR}\{73\}$ & & 1 & 1 \\
\hline $\mathrm{CR}\{83\}$ & & 1 & 2 \\
\hline $\mathrm{CR}\{84\}$ & & 2 & 1 \\
\hline $\mathrm{CR}\{85\}$ & & 1 & 1 \\
\hline $\operatorname{CR}\{115\}$ & & 4 & \\
\hline $\mathrm{CR}\{130\}$ & & 2 & 1 \\
\hline $\mathrm{CR}\{134\}$ & & 1 & 1 \\
\hline \multirow[t]{2}{*}{$\mathrm{CR}\{135\}$} & & 1 & 1 \\
\hline & CR $\{136\}$ (tambour + hochet) & & 1 \\
\hline
\end{tabular}

FIG. 5 - Nombre d'instrumentistes, types d'instruments et nombre de danseurs par œuvre.

\section{L'GuVre de Zacharias W WGNer}

La première mention de l'œuvre de Wagner ${ }^{28}$ date de 1937 : « un Xangô ${ }^{29}$ du temps des Hollandais qui ne diffère pas beaucoup de ceux d'aujourd'hui » (Gonsalves de Mello 2001 [1947], p. 221). Cette interprétation rapide fut assez largement reprise, en particulier par Ribeiro (cité in Tinhorão 2008 [1988], p. 35).

La vie aventureuse - et extraordinaire - de Zacharias Wagner (né à Dresde en 1614) a été reconstituée et commentée par Michel (1987) : celui que les Japonais allaient plus tard appeler l'« homme-tonnerre ", du fait d'un caractère " sévère et rude », est arrivé au Brésil en 1634, comme soldat de la WIC. Peu de temps après, il est devenu « écrivain des rôles militaires » ${ }^{30}$. L'auteur ne donne pas d'informations substantielles sur les activités du peintre pendant son séjour (une page en tout pour huit ans). Ce dernier a quitté le Brésil le $1^{\mathrm{er}}$ avril 1641, puis a été, toujours avec les Hollandais, au Cap, à Java et à Nagasaki, avant de revenir en Europe et d'y mourir en 1668. 


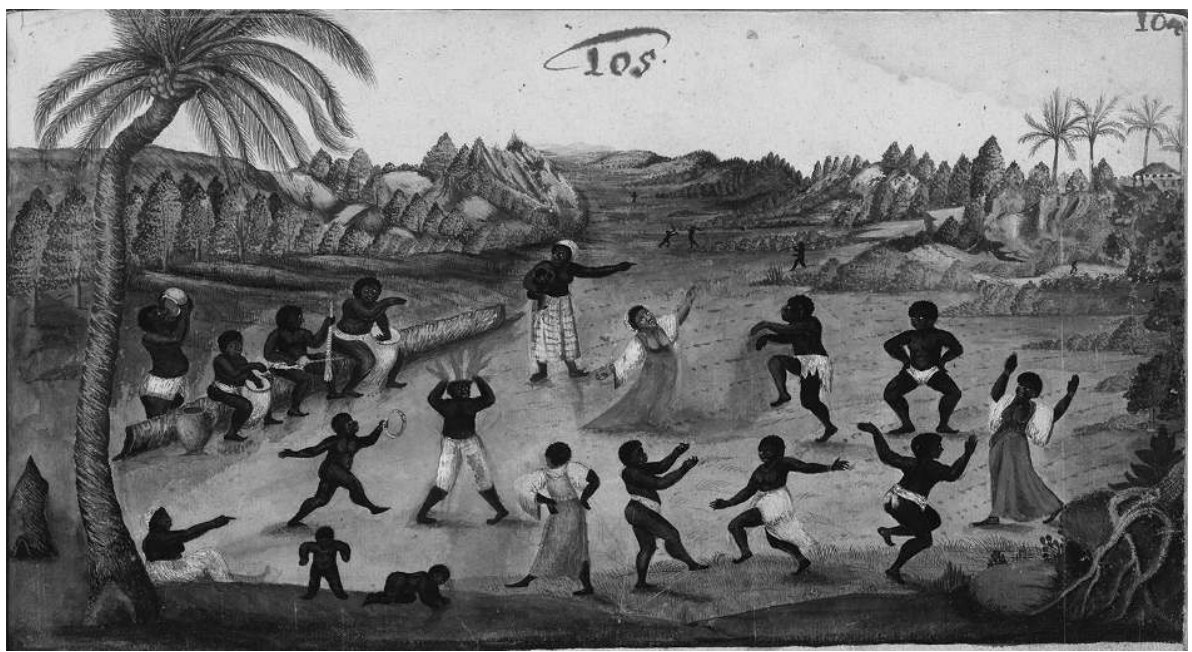

FIG. 6 et VI dans le cahier en couleurs-Zacharias Wagner. Negertanz, ca 1638. Gouache. 36 x $21,5 \mathrm{~cm}$. Kupferstichkabinett, Dresde.

L'œuvre de Wagner (Figure 6 et VI dans le cahier en couleurs), une gouache, présente la scène complète d'une forme musico-chorégraphique afro-brésilienne : c'est la première de l'époque coloniale, et l'une des seules de cette qualité documentaire. Trois musiciens assis sur un tronc couché (deux tambours assez ventrus, et un racleur très proche d'une bage ${ }^{31}$ contemporaine) font danser dix personnages (trois femmes habillées, un homme, et six autres à moitié dévêtus, qui pourraient être des adolescent[tes]). L'un d'entre eux agite un hochet cylindrique, de type tambourin à cymbalettes sans peau, qui est le seul instrument d'origine européenne (sans doute portugaise). À côté des musiciens, un personnage boit devant une jarre posée près du banc : l'association de la musique et de la boisson reste encore aujourd'hui très fréquente dans les formes afro-brésiliennes du Nordeste. Les tambours sont de tailles différentes, ce qui correspond au cas général dans les musiques africaines et/ou afro-américaines. Le personnage qui ajuste sa coiffe de plumes est unique dans les œuvres de l'époque hollandaise ; elle pourrait être interprétée comme une marque des interactions entre Noirs et Amérindiens, car les esclaves noirs ne sont, à cette seule exception, jamais représentés avec des ornements de plumes. Les Tupis côtiers étaient encore très présents au Pernambouc, et Post n'a pas manqué de souvent les peindre.

La précision du trait est moins habile que chez Post, mais les postures des corps rendent bien le mouvement dansé, tout comme les gestes des musiciens sont précis. On remarquera, par exemple, la main gauche posée à l'envers sur la peau du tambour, ce qui correspond à une technique utilisée ${ }^{32}$ pour produire un son étouffé, avant la frappe de la main droite qui est bien levée. C'est bien la preuve 
d'une observation très fine - et certainement répétée - de Wagner. Le texte écrit au verso peut être traduit ainsi :

Quand les esclaves terminent leur dure semaine de travail, ils reçoivent la permission de profiter selon leur goût de leur dimanche, quand, en se réunissant dans des lieux déterminés, ils dansent sans fatigue du matin au soir, avec les sauts et les contorsions du corps les plus variés, au son de tambours et de fifres joués avec beaucoup d'adresse, hommes et femmes, jeunes et vieux, au milieu de la plus grande confusion, pendant que d'autres marchent en rond en buvant une forte boisson faite de sucre appelée grappe ; de la même façon ils passent certains jours saints en dansant sans fin et finissent si sales et pleins de poussière qu'ils deviennent parfois méconnaissables. (d'après Tinhorão 2000, p. 57)

Wagner a également donné des éléments de description de funérailles, où les Noirs chantent en chœur devant le cercueil : « Ei, ei, ei, pourquoi es-tu mort ? Ei, ei, ei, est-ce qu'il t'a manqué du pain, de la farine de manioc, du tabac ou une pipe ? », et reviennent de la sépulture « en chantant et en dansant » ${ }^{33}$ (Wagner in Cabral de Mello 2010, p. 280). Il est intéressant de noter que le texte de Wagner, au dos de sa peinture, mentionne "des tambours et des fifres", alors que le tableau ne représente que des percussions. Sans doute l'auteur évoque-t-il d'autres formes dont il fut le témoin : on en trouve un écho dans les pratiques pernamboucaines contemporaines puisque la banda cabaçal, ou banda de pifano, qui anime (ou animait il y a peu) les bals populaires de forró est justement constituée de fifres et tambour ${ }^{34}$.

Cette œuvre isolée de Wagner constitue, en quelque sorte, un contrepoint à celles de Post : des qualités picturales et esthétiques bien moindres, mais une représentation moins conformiste et redondante que le motif postien. Le sujet présenté par Wagner est bien centré sur la forme afro-brésilienne en tant que telle. Et, comme on l'a déjà noté, il est envisageable de considérer qu'il s'agit d'une forme religieuse, même si le sujet est également proche d'un coco contemporain. Ce document revêt une importance inégalée comme source pour l'histoire de la musique brésilienne.

\section{QUELQUES REMARQUES ORGANOLOGIQUES}

$\mathrm{Au}$ Siècle d'or, la représentation des instruments de musique et, nous ajouterons, celle des positions de jeu, fait l'objet d'un réalisme certain, comme le montre le corpus présenté dans Buijsen et Grijp (1994). Pour l'étude de l'organologie, les œuvres pictographiques sont une riche source de documentation. Ainsi, Gétreau note, à propos des natures mortes représentant des instruments :

Elles constituent ainsi un réservoir d'informations particulièrement riche pour suivre l'évolution de la facture instrumentale. En comparant ces témoignages visuels avec les 
instruments réels conservés, on peut observer de nombreux détails fonctionnels symptomatiques du stade d'évolution d'un instrument ou d'un accessoire [...]. Quelques exemples d'œuvres au rendu quasi photographique ont même permis de reconnaître jusqu'au travail d'un atelier ou d'un facteur d'instrument. (2009, p. 15)

Dans son œuvre, Post peint essentiellement des tambours, qui sont tous du même type : unimembranophones coniques. On ne distingue pas de douves, donc tout porte à croire qu'ils sont monoxyles (sans doute creusés dans un tronc par la main de l'homme ou, préalablement, par des insectes). On ne peut donc se permettre de poser l'hypothèse de la fabrication précoce de tambours construits en douves de tonneaux, une technique qui a connu un grand succès au $\mathrm{xx}^{\mathrm{e}}$ siècle pour les tambours afro-américains. Ils sont portés en bandoulière, du côté gauche du corps. Cela correspond à une observation précise, car c'est bien la manière la plus commode de porter l'instrument pour un droitier.

Dans le tableau de Wagner, les tambours sont assez proches des tumbadoras cubaines et, plus encore, des tamboriles afro-uruguayens. Ces tambours semblent en lien avec les tambours bantous de type ngoma. Le mode de fixation de la peau est du type collé ou lié par une corde : il n'apparaît pas de chevilles ou de système de laçage comme c'est fréquemment le cas en Afrique de l'ouest. Cela est cohérent avec la provenance des esclaves qui venaient très largement de la région du Congo et de l'Angola.

Les tambours représentés par Post ont une descendance, directe ou, plus probablement, indirecte : on citera, parmi les plus courants, l'atabaque (Figure 7) et le timbau. Le premier est utilisé dans les religions afro-brésiliennes (candomblé, umbanda, etc.) et les rondes de capoeira ${ }^{35}$. Il existe aujourd'hui à Olinda un lieu de culte (Nação Xamba) qui utilise des tambours du même type, appelés en portugais engome, déclinaison brésilienne du terme bantou ngoma évoqué plus haut. Le timbau, de (ré)invention récente, est un tambour conique à la peau synthétique très tendue, servant de tambour soliste, joué debout et avec des phrasés parfois proches des djembés africains, dans des musiques de percussion modernes, comme la timbalada ${ }^{36}$. À Récife, il est parfois joué dans le maracatu ${ }^{37}$.

\section{Présence de la musique et de la danse dans l'oeuvre de Post}

C'est à partir de sa seconde période (1645-1659), appelée les «années réalistes » par Corrêa do Lago et Ducos (2005, p. 22) que Post présente fréquemment le motif musical que nous avons défini. Cette dimension sonore ajoutée en quelque sorte au tableau - les instruments sont joués, les corps en mouvement -, donne une nouvelle intensité et une proximité aux scènes représentées. Comme le note très justement Leppert (1993, p. 29) : "Sound, by its enveloping character, brings us closer to everything alive." 


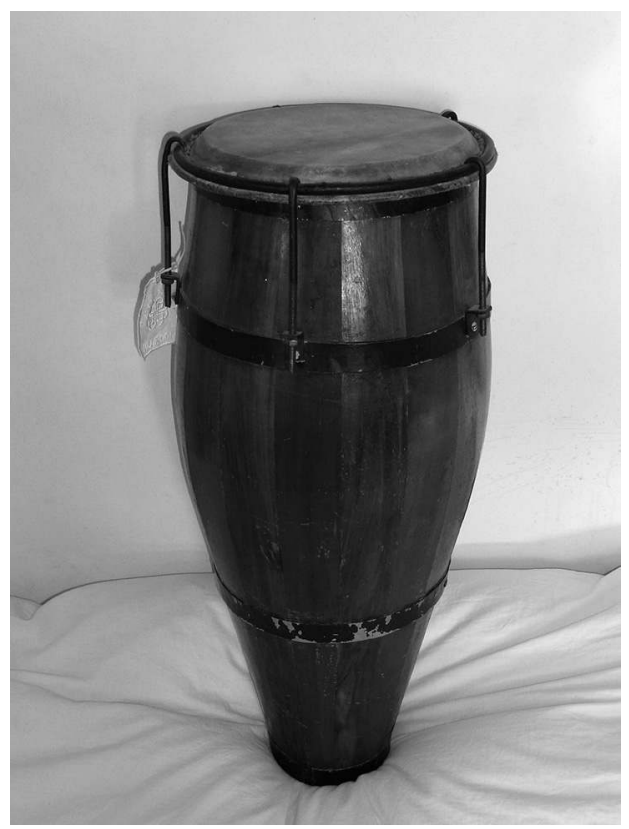

FIG. 7 - Tambour unimembranophone conique contemporain : atabaque lê du candomblé de Bahia (cliché Estival).

Alors que les tableaux de cette période étaient sans doute destinés à des clients qui participèrent à l'aventure coloniale hollandaise, il est intéressant de noter que la représentation de musiciens et danseurs peut aussi être considérée comme un marqueur mnésique : le son puissant (et inconnu, sous cette forme, des NordEuropéens) des tambours a dû s'imprimer dans l'espace sonore colonial et, donc, dans les mémoires auditives hollandaises. La présence dans l'œuvre de scènes musicales, par l'évocation des sons qu'elle suscite, facilite le processus de remémoration, et sans doute une certaine saudade ${ }^{38}$, auprès des clients potentiels de l'artiste.

Dans les paysages lointains, on ne distingue pas de figuration de scène musicale et chorégraphique. Dans les représentations consacrées au monde du travail, une seule œuvre met en scène des musiciens près d'un moulin à sucre, et ce dernier n'est pas en fonctionnement. L'ambiance générale des tableaux avec musique et danse est calme, pacifique; cela nous amène aux trois remarques suivantes, qui nous laissent entrevoir la distance entre les représentations proposées par les œuvres et la réalité brésilienne de l'époque :

- le climat, et plus généralement les espaces naturels ou humanisés, sont peints sous un jour serein, sans vents violents ou inondations, sans soleil 
écrasant ${ }^{39}$ et sans feux (de déforestation, d'essartage, de récolte de canne ou d'incendies) ;

- les conflits qui émaillèrent la période coloniale hollandaise sont absents des œuvres, alors que la paix ne fut jamais réellement établie. Le journal du mercenaire alsacien Ambrosius Richshoffer (2004 [1677]), comme les descriptions de la vie coloniale compilées par Gonsalves de Mello (2001 [1947]) ou de Cabral de Mello (2010) relatent une dure réalité que l'on peine à reconnaître dans les œuvres de Post. Il est même particulièrement frappant de constater que les représentations de soldats les montrent dormant au pied d'un arbre (CR $\{6\}$ ), ou accompagnant les autorités (CR $\{22\})$ alors que les actions militaires contre les révoltés portugais ou, à tout le moins, les escarmouches furent presque permanentes ;

- le travail dans les usines à sucre (CR \{47, 48, 50, 54, 57, 58, 59\}) paraît presque harmonieux, les scènes de vie quotidienne sont paisibles et ne reflètent en rien la grande violence sociale qui marqua cette aventure coloniale. Cette société esclavagiste, comme les autres, était particulièrement dure, cruelle et injuste pour les captifs. Pierre Moreau a décrit les conditions de travail terribles et les châtiments que subirent les Noirs dans la colonie brésilienne, et prévient le lecteur de ces horreurs : " J'appréhende quasi la façon inhumaine et impitoyable dont on use envers ces malheureux captifs, puisqu'elle va au-delà de la compassion, \& excite le frémissement. » (1651, p. 40.)

Ainsi, les choix de Post relèvent sans doute de ce que Leppert, après Winternitz, appelait la " prettification ", dans le contexte économique particulièrement concurrentiel du Siècle d'or hollandais : " And since people do not generally buy art which offends their sensibilities or is ugly in its realism, it naturally would seem wise for the astute artist to paint his pictures in a manner appropriate to the tastes, perhaps even values, of his patrons. ॥ $(1975$, p. 4.)

Schama (1987) a montré que, dans l'œuvre de Jan Steen, et de ses contemporains, "Money, as well as sex, was something of a Dutch fixation [...] ». Chez Post, aucun caractère lascif, ou à connotation sexuelle, n'est présent dans les représentations de la musique et de la danse. Ce thème est pourtant un classique des chroniqueurs qui ont évoqué les formes afro-américaines (voir les sources écrites, mais aussi Labat 1993 [1698]). Peu de représentations religieuses non plus : les églises sont de simples éléments d'urbanisme dans les paysages. Alors que les Portugais catholiques ont exercé - ou tenté d'exercer - au Brésil un considérable contrôle social et religieux sur les esclaves, les Néerlandais n'ont jamais vraiment appliqué de politique de christianisation. Une lettre du Conseil des XIX, datée de 1635, va pourtant dans ce sens, mais elle n'a jamais été suivie d'effets systématiques. Selon la synthèse de J. A. G. de Mello (2001 [1947], p. 198), les pasteurs se sont surtout occupés de la moralisation de la vie des Européens, ce qui n'était pas une mince affaire. 
On remarquera enfin que, dans le cadre d'un projet de construction d'un exotisme colonial naissant, la musique des populations d'origine européenne n'est jamais représentée. On sait pourtant que les Portugais, comme les Hollandais et leurs mercenaires, avaient des activités festives et musicales, même si la documentation à leur sujet est assez rare dans les sources publiées et analysées. Par exemple, concernant les mercenaires français de la VOC ${ }^{40}$, nous disposons du court témoignage contemporain de Jean Guidon de Chambelle à propos de la fête donnée à l'occasion de l'arrivée d'un nouveau gouverneur de Batavia en 1646 :

Après avoir bu chacun une fois ou deux, nous prîmes pour armes des branches de palmes et sur la tête des couronnes de palmes, et allâmes visiter les autres compagnies, ayant notre étendard, tous en ordre, au son de quelque flûte et violons, les uns dansant, les autres sautant et faisant mille bouffonneries. (2003 [ms. XVII ${ }^{\mathrm{e}}$ siècle], p. 136)

On ne trouvera pas de représentation de ce type de réjouissance dans l'œuvre de Post. Pourtant, des peintres contemporains comme Jan Steen, Gerrit Dou, David Teniers le Jeune, dans un souci sans doute plus moralisateur que documentaire, firent de la musique et de la danse de leurs compatriotes des thèmes importants de scènes de genre qui sont restées célèbres jusqu'à nos jours. Les Européens sont parfois présents $(\mathrm{CR}\{42\},\{115\})$ dans les scènes paysagères de Post, mais ils ne prêtent pas ostensiblement attention aux danses afrobrésiliennes qui sont exécutées devant eux. Comme ce fut le cas général dans l'empire colonial néerlandais, la séparation des populations était stricte. Les Amérindiens, les Européens et les esclaves d'origine africaine ne se cotoyaient que dans des relations de domination liées à la sphère économique, ou parfois militaire, ce qui amena Gilberto Freyre à évoquer de façon synthétique et caricaturale « une minorité de blonds exploitant et dominant un prolétariat de gens de couleur » (cité in Gonsalves de Mello 2001 [1947], p. 199).

Post, comme Wagner, a certainement été le témoin oculaire (et auditif) direct des pratiques musicales qu'il illustre. Post, peintre paysager, a beaucoup voyagé dans le Brésil hollandais et a croqué les scènes qu'il décrit. Dans la composition des tableaux, le motif postien est généralement proche de - ou contigu à d'autres motifs types, comme par exemple des personnages portant des paniers, en conversation, ou des Indiens avec leurs arcs et flèches, etc. Les attitudes sont précises et plausibles, tout comme le sont les remarquables représentations de moulin à manioc (CR $\{14\})$ ou d'usine à sucre. On a vu qu'il serait illusoire d'interpréter les tableaux hollandais du Siècle d'or comme de simples photographies de la réalité. Il nous faut cependant considérer que, dans leur immense majorité, les œuvres sont des représentations fidèles d'éléments de paysages, de scènes de vie quotidienne prises sur le vif, de documents à valeur économique, au cœur d'un projet unique au XVII ${ }^{\mathrm{e}}$ siècle : "Post's apparent artlessness in framing his compositions without regard to conventional landscape tradition is exemplified in all his Brazilian paintings. " (Gordon 2007, p. 67.) 
C'est l'agencement des motifs, la composition du tableau, qui relèvent d'une construction esthétique où se (re)crée un nouveau monde (selon l'expression d'Oliveira 2005a, p. 8) ; et, bien sûr, malgré son originalité (thématique, esthétique), Post est bien un Hollandais du XVII ${ }^{\mathrm{e}}$ siècle qui peint pour une clientèle de Hollandais du XVII ${ }^{\mathrm{e}}$ siècle... Prenons un exemple concret : le tableau CR $\{84\}$ (Figure 8 et VII dans le cahier en couleurs).

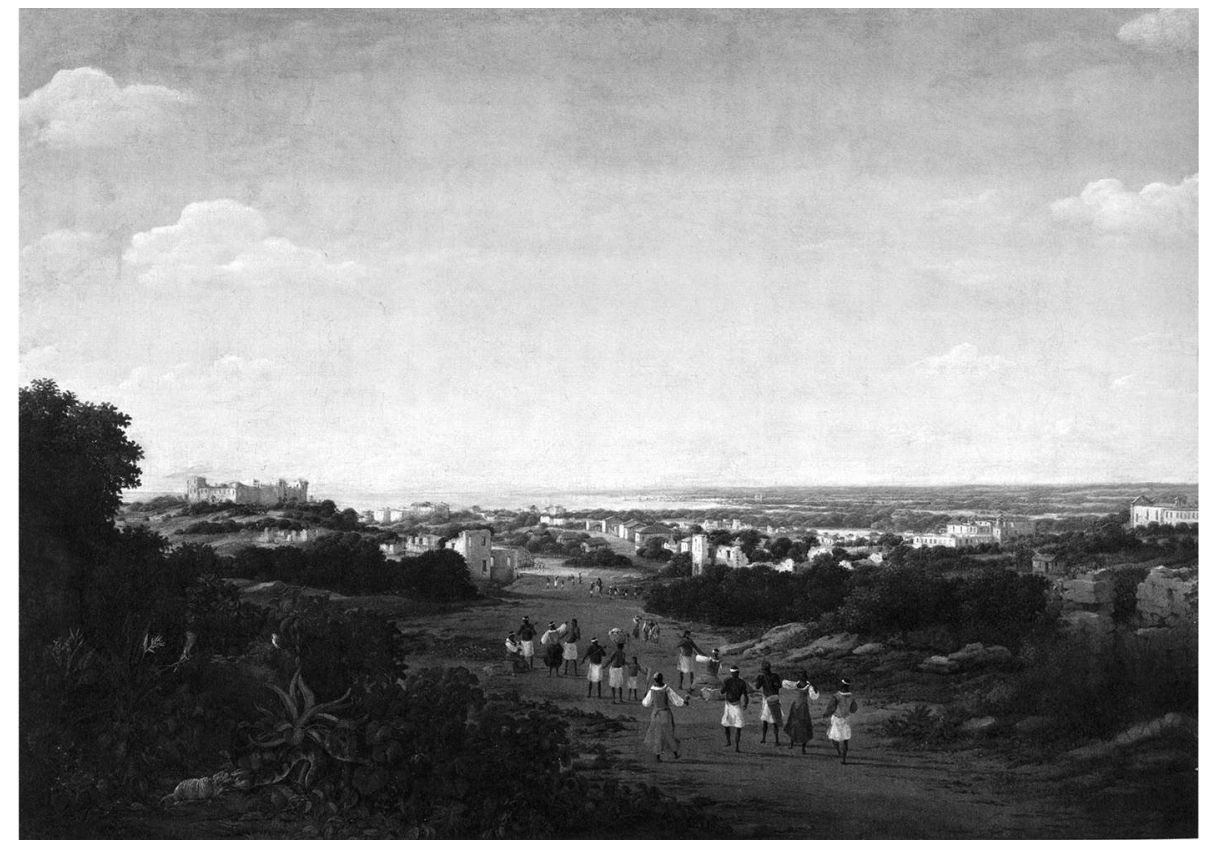

FIG. 8 et VII dans le cahier en couleurs - CR \{84\} Frans J. Post. Vue des ruines d'Olinda, ca 1665 . Huile sur toile. 77 x $110 \mathrm{~cm}$. Museu Nacional de Belas Artes, Rio de Janeiro.

Cette œuvre présente, au premier plan, un repoussoir. Il comprend de la végétation et des animaux où l'on peut identifier : une Agavaceae, un perroquet, un autre oiseau, et un boa commençant à dévorer un oppossum. Leur assemblage relève très clairement de la composition du tableau, et ne peut, en aucun cas, correspondre à une réalité zoologique in situ. Les oiseaux, l'opossum et le reptile sont pourtant assez bien peints ; la précision topographique, avec la perspective de l'île de Récife et les deux tours blanches du palais de Jean-Maurice de Nassau est bonne. Le groupe de musiciens et danseurs, en position à peu près centrale dans la partie terrestre du tableau (le ciel occupe les deux tiers de l'œuvre), est placé sur un large chemin, ou une partie de terrain vague, qui se prolonge sur une diagonale. Si l'on se concentre sur ce groupe, on reconnaît bien le motif principal, 
avec un tambourinaire torse nu, vu de face, et deux couples de danseurs plus au premier plan. Les tableaux $\mathrm{CR}\{83\}$ et $\mathrm{CR}\{85\}$, ayant le même titre, sont similaires à $C R\{84\}$, ce qui montre que le peintre n'hésitait pas, sur une période courte, à reprendre des motifs qui s'intégraient bien dans la composition. On peut imaginer que, vers 1665 (Corrêa do Lago et Corrêa do Lago 2007, p. 248), Post a pu avoir trois commandes d'une œuvre qui plaisait, car elle associait des éléments paysagers et exotiques (dont les animaux et notre motif), caractéristiques de l'aventure brésilienne.

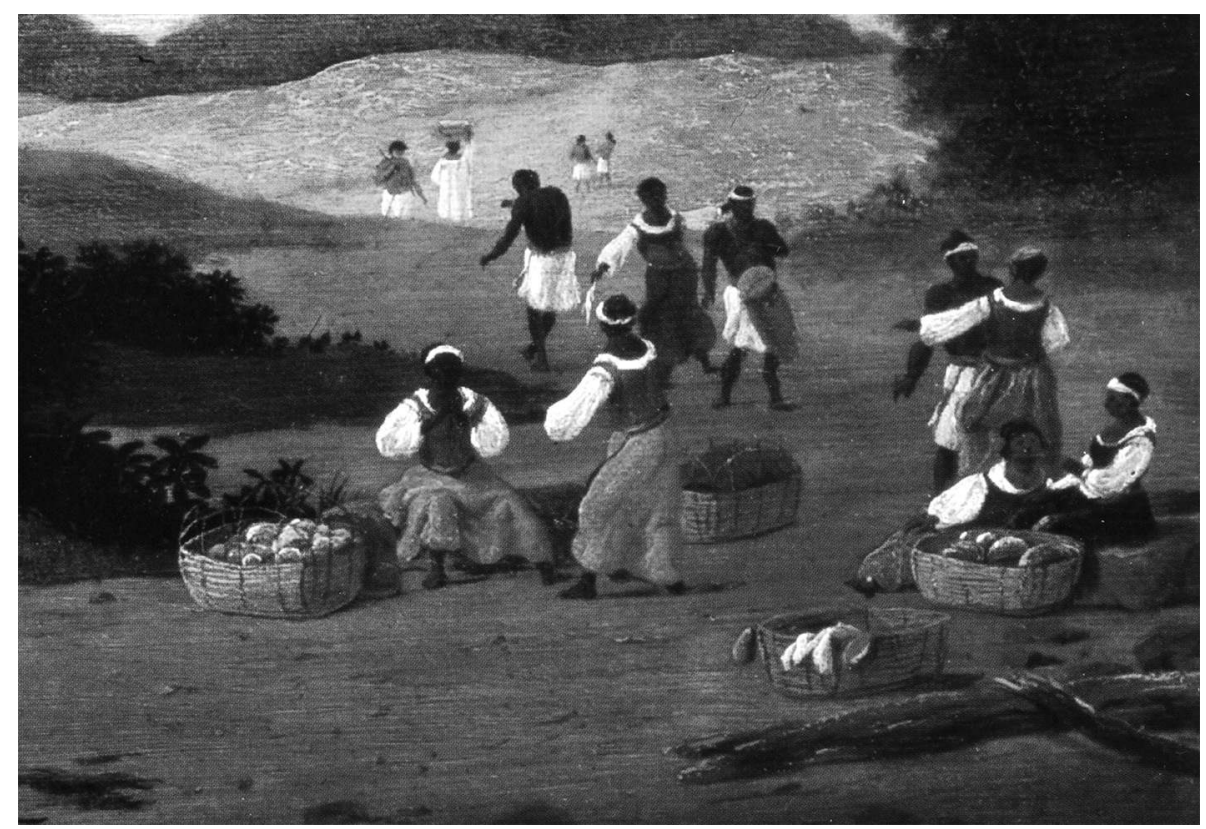

FIG. 9 et VIII dans le cahier en couleurs - Détail de CR \{26\} Frans J. Post. Maison de planteur, 1655. Huile sur toile. $46.5 \times 62.9 \mathrm{~cm}$. Los Angeles County Museum of Art, Los Angeles.

Un autre exemple : le très beau tableau CR $\{26\}$ (Figure 9 et VIII dans le cahier en couleurs) présente un danseur, derrière le tambourinaire et une danseuse, dont le geste retiendra l'attention de quiconque a voyagé de nos jours au Brésil : la position du corps et le déplacement que suggère de façon magistrale la représentation du danseur correspond au mouvement basique de la capoeira, appelé ginga. Il s'agit du mouvement de base, balancement latéral des bras simultané à un changement d'appui avant-arrière des jambes. Il n'y a certes pas dans cette œuvre de Post d'évocation de la lutte dans une ronde de capoeira mais l'homologie du mouvement dansé est manifeste. Cette plongée dans le passé et 
dans l'histoire des corps afro-américains n'est sans doute qu'une trace, mais elle atteste une probable permanence troublante. Le motif postien de la musique et de la danse est en général représenté comme un élément public du paysage : il ne s'agit pas de cérémonie importante, ou de procession, mais de petit(s) groupe(s). Les musiciens et danseurs voient leur performance représentée dans des espaces libres du tableau, qui sont généralement des espaces intermédiaires de la topographie : terrains vagues, bordures de routes ou de chemins. On peut donc penser qu'il s'agit de performances assez informelles, d'éléments de fêtes ou de cérémonies de petite échelle, hors des cadres contraints ou processionnels qui pouvaient rythmer le calendrier de la colonie. Musiques et danses intimes en quelque sorte, qui sont aussi représentées parce qu'elles attestent de la connaissance, elle aussi intime, qu'a pu avoir Post de l'aventure brésilienne hollandaise.

Pourtant, notre motif n'apparaît pas dans les sources écrites consultées, et nous pouvons nous interroger à ce propos : les chroniqueurs mentionnent des Noirs jouant la musique des maîtres, ou participant à des processions, ou encore réalisant des chants et danses manifestement liées aux réminiscences des religions africaines introduites avec eux au Nouveau Monde. Ces chroniques ne mentionnent en réalité que de façon marginale la musique et la danse, et l'écriture de ces textes ne relève pas de la même logique que la composition picturale ; ils ont aussi été produits par des voyageurs de passage, qui n'avaient sans doute ni le temps ni le loisir d'assister à des formes de la vie courante telles que celles représentées par Post. Une hypothèse plausible serait que nos artistes aient privilégié, parmi l'ensemble des séances de musique et danse auxquelles ils ont pu assister lors de leur long séjour au Brésil, celles qui étaient les plus éloignées des européennes connues et des instruments habituels de l'Ancien Monde. Cela leur a permis de conforter l'exotisme de leurs œuvres et, ainsi, d'en accroître la valeur, tant sur le plan commercial que sur le plan documentaire. Pour la gouache de Wagner, il deviendrait alors plus compréhensible que le texte au dos de l'œuvre mentionne des tambours et fifres, alors que sont représentés au recto tambours unimembranophones et racleur.

\section{Conclusion}

Si le monde sonore du Brésil du XVII ${ }^{\mathrm{e}}$ siècle nous restera à jamais inaudible, les œuvres de Post et de Wagner sont des documents irremplaçables en ce qui concerne l'histoire des musiques noires dans ce pays et, plus largement, dans les Amériques. La musique et la danse, par un motif récurrent, peuplent nombre des paysages peints par Post ; les instruments, les attitudes, les positions de jeu comme les gestes chorégraphiques sont le fruit d'une knowledge by acquaintance, et relèvent d'une observation précise, et très certainement répétée de la part du peintre. En cela, comme ses célèbres compatriotes contemporains Steen ou 
Teniers le Jeune, Post représente la musique comme il l'a vue et entendue, et en intègre des images dans ses œuvres, sans que la finalité d'une représentation documentaire globale ne soit première : l'artiste a conçu un motif type, qu'il a placé, comme d'autres (humains ou éléments naturels remarquables), dans la composition de ses tableaux ; celle-ci met en valeur les éléments exotiques, prisés des clients du peintre.

Le motif postien peut alors être considéré comme une trace, laissée dans les tableaux à l'image d'un matériel dans une couche archéologique. Il est impossible de décider si le motif représenté correspond à une reconstruction, dans l'Amérique du XVII ${ }^{\mathrm{e}}$ siècle, d'une forme africaine, ou à l'un des premiers éléments du métissage musical inter-africain et africain/européen qui allait avoir une si riche descendance au Brésil. Si, comme c'est probable, le tambour accompagnait la voix humaine, celle-ci s'est définitivement tue.

Wagner, avec un trait moins sûr, et un métier bien moindre a, quant à lui, donné à voir ce qui constitue aujourd'hui encore, une des formes canoniques des genres musicaux et chorégraphiques brésiliens : tambours, idiophone, danse et, très certainement, voix humaine. Les œuvres dont nous avons esquissé ici l'analyse donnent incontestablement une profondeur historique certaine aux formes afro-brésiliennes. Comme nous l'avons évoqué au début de cet article, les trois siècles suivants, tant par l'iconographie que par les textes, ne nous ont guère fourni d'éléments informatifs plus précis. Il faudra attendre les enquêtes d'Andrade à la fin des années 1920 (2002) et la Missão de pesquisas folclóricas qu'il diligenta en 1938 pour que des éléments documentaires robustes soient enfin rassemblés sur les musiques populaires du Nordeste brésilien.

Enfin, le motif postien pourrait bien avoir une descendance, lointaine et certainement indirecte, dans la grande famille des coco, genre musicochorégraphique très répandu dans la Zona de Mata et sur le littoral du Pernambouc et du Paraiba (Ayala et Ayala 2000; Sobrinho 2006). Aujourd'hui, les groupes de coco, souvent constitués d'un(e) chanteur(euse)/compositeur(trice) et de ses accompagnateurs/percussionnistes, se produisent pendant les fêtes des saints populaires (festas juninas) comme Santo Antônio, São João, São Pedro, Sant'Ana et sur les diverses scènes programmant des formes traditionnelles. Il n'en reste pas moins que de nombreux moments informels permettent de voir et de participer à des coco dans les communautés mêmes dont ils sont originaires, comme Itamaracá, Itapissuma, Igarassu. Et c'est là, sans doute, que les résonances avec le motif postien sont les plus sensibles : au moment de la Saint-Jean, il n'est pas rare de voir des groupes pratiquer ou s'entraîner dans des lieux aussi improbables que ceux qu'a meublés Post avec son motif (Figure 10). *

* Manuscrit reçu en juillet 2013, accepté pour publication en février 2014. 


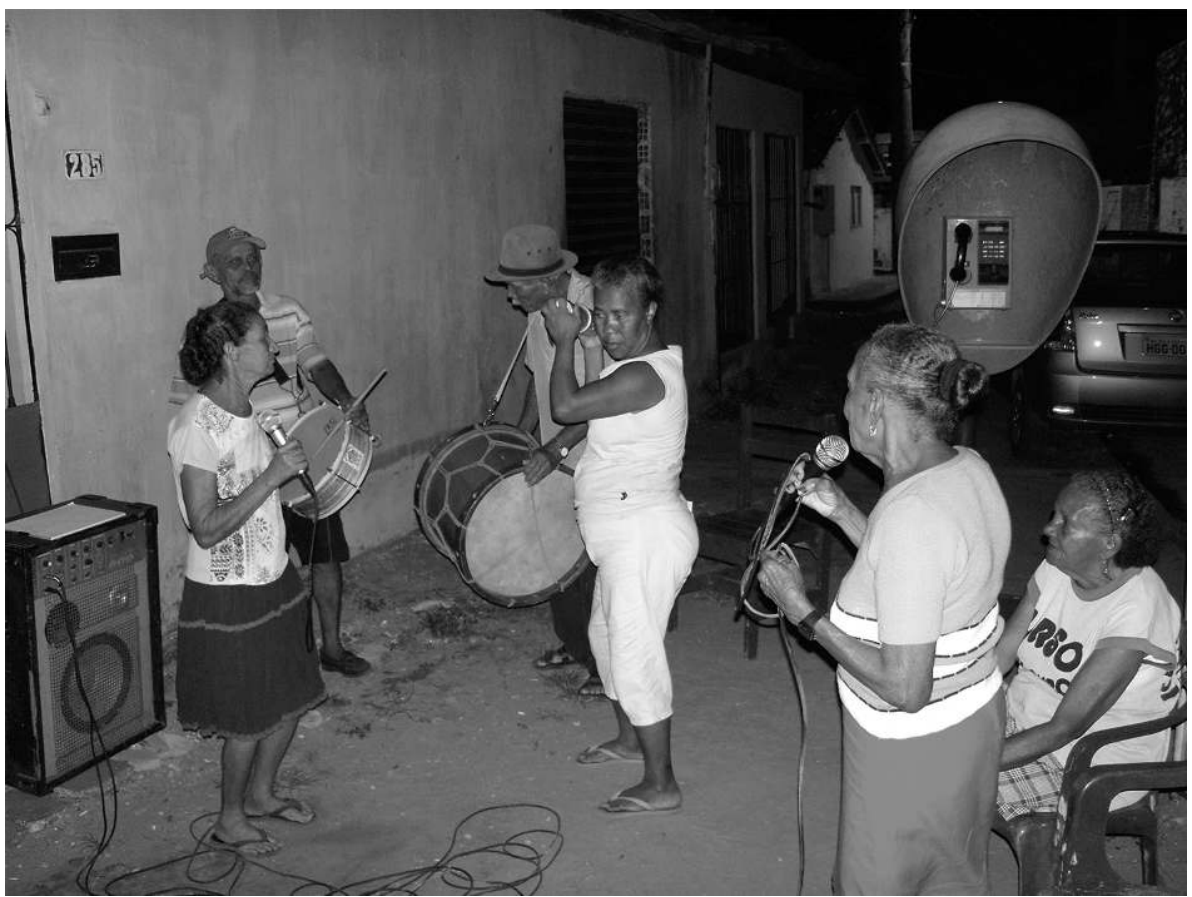

FIG. 10 - Un coco informel à Itapissuma en 2009 (cliché Estival).

\section{NOTES}

1. La Hollande est l'une des entités de la république des Provinces-Unies des Pays-Bas (comme on désignait le pays au $\mathrm{XVII}^{\mathrm{e}}$ siècle); par métonymie, en français comme en portugais, on désigne fréquemment par le terme « Hollande » l'ensemble de la confédération.

2. La république des Provinces-Unies ne sera officiellement reconnue par l'Espagne qu'en 1648.

3. Johan Maurits van Nassau-Siegen (1604-1679), comte d'origine allemande, filleul du gouverneur général (stadhouter) de Hollande et de Zélande Maurice de Nassau, était parent de plusieurs monarques d'Europe, dont Louis XIV.

4. Poète et compositeur, il fut l'un des importants intellectuels du XVII ${ }^{\mathrm{e}}$ siècle. Il était l'ami de René Descartes et de Rembrandt van Rijn.

5. Les Néerlandais, protestants, comme leurs mercenaires allemands ou français, surent - au moins pendant le gouvernement de Jean-Maurice de Nassau - cohabiter en assez bons termes sur le plan religieux avec les nombreux juifs (hollandais, mais souvent d'origine portugaise) et les planteurs portugais catholiques de la campagne sucrière.

6. Les tableaux furent exposés à Versailles, et vus par le roi en août 1679. Pour les données historiques sur les œuvres en France, les circonstances de leur don et leur devenir, on se référera à Corrêa do Lago et Ducos (2005, pp. 29-35). Une rétrospective leur fut consacrée en 2005-2006 au Louvre : « Frans Post : le Brésil à la cour de Louis XIV » (commissariat de Pedro Corrêa do Lago et Blaise Ducos).

7. Son frère Pieter Post (1608-1669) fut un architecte (et aussi un peintre et un dessinateur) de grand renom (il dessina, entre autres, les plans de la Mauritshuis à La Haye, pour Jean-Maurice de Nassau). 
8. Ces tableaux font partie du don fait à Louis XIV. Il en reste 7, considérés par Corrêa do Lago comme la «première phase » du peintre.

9. L'indépendance des Provinces-Unies fut obtenue de fait en 1581, mais l'Espagne ne la reconnut officiellement qu'avec le traité de Westphalie (1648).

10. Kyrova 1994, p. 31.

11. Ibid., p. 32.

12. Il est vrai que la musicologie historique ne s'intéresse guère plus aux musiques populaires européennes anciennes, sans doute faute de sources.

13. Aujourd'hui la ville de Récife. Parana buka ou Parana puku (du tupi de la côte « où se brise la mer »/«longue mer », étymologies sujettes à caution...) fut aussi agréablement écrit en français «Fernanbourg » jusqu'à la fin du XvII ${ }^{\mathrm{e}}$ siècle...

14. Tinhorão 2000, p. 80, citant un ata da Câmara de Salvador de 22 de novembro de 1673 (« édit de la chambre municipale de Salvador $»$ ).

15. Tinhorão, 2000, p. 89, citant Francisco Manuel de Melo ( $c a$ 1658).

16. En portugais : charamelas, caixas et trombetas.

17. En portugais : buzinas, flautas et atabaques. Le terme buzina a la définition suivante dans le dictionnaire de référence Aurélio : « nom commun à divers types de trompes de corne ou de métal qui produisent un son unique, fort ». Atabaque est défini comme : « espèce de tambour avec une peau d'un seul côté et percuté avec les mains » [traductions de l'auteur, J.-P. E.].

18. Tinhorão 2008 [1988], pp. 37-42.

19. Ou lundu. Le mot a varié au cours des siècles : le lundu est un genre musical métissé, harmonisé, aux $\mathrm{XIX}^{\mathrm{e}}$ et $\mathrm{Xx}^{\mathrm{e}}$ siècles. Il était sans doute, à ses origines, une forme musico-chorégraphique du même type que les batuques : musique et danse afro-brésiliennes où prédominent les voix et les tambours, en lien avec les dieux africains éponymes.

20. Post a vraisemblablement dessiné des centaines, voire des milliers de croquis au Brésil (Corrêa do Lago et Corrêa do Lago 2007, p. 371).

21. Comme $\mathrm{CR}\{76\}$, où deux personnages semblent danser face à face, sans la présence d'aucun instrument.

22. Il est très difficile, en tout cas aujourd'hui, de différencier les Portugais des Nord-Européens (Néerlandais et leurs mercenaires), sauf lorsqu'on a des contextes explicites (CR $\{22\}$ par exemple) représentants le pouvoir politique ou ses soldats.

23. Au Brésil, les calebasses sont généralement secouées : elles sont, soit à percussion interne par de tous petits objets durs (type maraca amérindienne), soit à frottement externe par un filet de perles (type abê utilisés dans le Xangôo).

24. Cette hypothèse nous semble la plus vraisemblable, car les calebasses sont fragiles à la percussion et se rompent facilement dès qu'on les frappe avec un objet d'une certaine masse, ou qu'elles tombent par terre.

25. Elle figure également en couverture de l'ouvrage de Tinhorão (2008 [1988]).

26. Maracatu: procession carnavalesque typique de Récife et de sa région, mettant en scène une cour royale d'inspiration africaine dansant et déambulant au son d'un puissant orchestre composé d'au moins une quinzaine de tambours (alfaia, caixa), de hochets (mineiro) et de cloches (gonguê). D'un point de vue sociologique, les groupes de maracatu sont traditionnellement fortement territorialisés, et s'autodésignent généralement comme "nation », sans doute en référence aux anciennes confréries noires.

27. La croix originale a été cassée par un camion dans les années 1980.

28. Diverses graphies du patronyme ont été utilisées : «Wagener » dans une édition anglaise (1704), reprise au Brésil en 1964, « Wagenaar » en Hollande (1724), « Wagenaer » en afrikaans en Afrique du Sud en 1973 (il a été le second commandant du Cap). Il s'agit bien, malgré tout, du même personnage (voir Michel 1987, pp. 54-55).

29. Xangô désigne l'un des orixa (divinités) principaux du panthéon yoruba du Brésil (et de Cuba). À Récife, il désigne l'ensemble du culte afro-brésilien, assez proche du candomblé de Bahia. 
30. Le terme ancien Muster Schreiber est traduit en allemand moderne Schreiber der Musterrollen par Michel (1987, p. 84).

31. La bage, appelée aussi canzá, est un idiophone raclé d'une cinquantaine de centimètres de long, constitué d'un pièce de bambou ou de bois avec de multiples entailles transversales, comme dans le dessin de Wagner. Il est utilisé, au Pernambouc, dans l'orchestre de la forme de théâtre musical cavalo marinho (Murphy 2008).

32. Nous l'avons observée avec Iraquitã, maître-tambour d'un lieu de culte (terreiro Xamba Nagô) de la religion afro-brésilienne Xangô d'Olinda.

33. Traduction du portugais de l'auteur (J.-P. E.), l'original allemand ne nous ayant pas été accessible.

34. Précisément deux pifes (pifanos), fifres, un tambour grave joué avec deux baguettes différentes zabumba, un triangle et, parfois, une petite caisse claire appelée caixa de guerra ou tarol. Le forró est le genre emblématique des danses de couple du Nordeste brésilien. Il comprend des danses comme le baião, le xote (scottish), le xaxado, etc.

35. Capoeira : art martial dansé, d'origine afro-brésilienne, qui a connu dans la seconde moitié du $\mathrm{xx}^{\mathrm{e}}$ siècle un formidable succès : au Brésil tout d'abord, jusqu'à en devenir l'un des emblèmes, puis dans le monde entier.

36. L'orchestre Banda Timbalada, fondé par Carlinhos Brown dans les années 1980, en fut le promoteur. Brown considère qu'il a inventé l'instrument, en modifiant un atabaque plus ancien, joué transversalement dans le samba.

37. Maracatu Nação Porto Rico. Au moment de son introduction par ce groupe, il a été largement rejeté comme n'étant pas « traditionnel »...

38. On ne nous tiendra pas rigueur, nous espérons, de cet anachronisme brésilien...

39. C'est, en l'occurrence, bien sûr aussi pour des raisons artistiques, que Post a peint des ciels en général nuageux : cela facilite le traitement réaliste et documentaire des couleurs, et élimine le problème technique des ombres contrastées. À ce sujet, voir Gordon 2007, pp. 71 et 76-77.

40. Verenigde Oostindische Compagnie, cousine orientale et contemporaine de la WIC, dont les recrutements militaires étaient comparables.

\section{RÉFÉRENCES CITÉES}

AlPERs Svetlana

$1990 \quad$ L'art de dépeindre : la peinture hollandaise au XVII ${ }^{e}$ siècle, Gallimard, Paris.

ANDRADE Mário de

2002 Danças dramáticas do Brasil (édition organisée par Oneida Alvarenga), Editora Itatiaia, Belo Horizonte.

Ayala Maria Ignez Novais et Marcos Ayala (éd.)

2000 Côcos : Alegria e devoção, Editora da Universidade Federal do Rio Grande do Norte, Natal.

Barlaeus Caspar

1647 Rerum per octennium in Brasilia et alibi nuper gestarum, sub praefectura Illustrissimi Comitis I. Mauricii, Ioannes Blaue, Amsterdam.

BERGSTRÖM Ingvar

1956 Dutch still-life painting in the seventeenth century, T. Yoseloff, New York.

BOXER Charles Ralph

2004 Os Holandeses no Brasil, 1626-1654, Companhia Editora Nacional, Recife [1957]. 
BRIENEN Rebecca Parker

2006 Visions of savage paradise : Albert Eckhout, court painter in Colonial Dutch Brazil, 1637-1644, Amsterdam University Press, Amsterdam.

BUIJSEn Edwin et Louis Peter GriJP (éd.)

1994 The Hoogsteder exhibition of music and painting in the Golden Age, catalogue d'exposition, Hoogsteder \& Hoogsteder, La Haye.

Cabral de Mello Evaldo (éd.)

2010 O Brasil holandês, Penguin Classics, São Paulo.

CARNEIRo Edison

1982 "Samba de umbigada », in Folguedos tradicionais, Fundação Nacional de Artes, Rio de Janeiro [1961].

Charles-Dominique Luc

1994 Les ménétriers français sous l'Ancien Régime, Klincksieck, Paris.

CorrêA do LaGo Pedro et Blaise Ducos

2005 Frans Post, le Brésil à la cour de Louis XIV, catalogue d'exposition, Louvre Éditions/5 Continents, Paris.

CorrêA do Lago Pedro et Bia CorrêA do Lago (éd.)

2007 Frans Post (1612-1680). Catalogue raisonné, Louvre Éditions/5 Continents, Paris.

DUPARC Frederik J.

2012 Post : (2) Frans Post, Oxford University Press, Oxford [http://www.oxfordartonline.com/subscriber/article/grove/art/T068936pg2, consulté le 17/04/14].

FISCHER Peter

1972 "Music in paintings of the Low Countries in the sixteenth and seventeenth century », Sonorum Speculum, pp. 50-51.

GÉTREAU Florence

1997 Musiciens des rues de Paris, catalogue d'exposition, Éditions de la Réunion des Musées Nationaux, Paris.

2009 Voir la musique. Les sujets musicaux dans les æuvres d'art du XvI ${ }^{e}$ au $x X^{e}$ siècle, catalogue d'exposition, Musée départemental de l'abbaye de Saint-Riquier, Saint-Riquier.

Gonsalves de Mello José Antônio

2001 Tempo dos Flamengos. Influência da ocupação holandesa na vida e na cultura do norte do Brasil, Topbooks Editora, Rio de Janeiro [1947].

GoRDON George

2007 «Frans Post : style and technique », in Pedro Corrêa do Lago et Bia Corrêa do Lago (éd.), Frans Post (1612-1680). Catalogue raisonné, Louvre Éditions/ 5 Continents, Paris.

Guidon de Chambelle Jean

2003 «Voyage des Grandes Indes orientales », in Mercenaires français de la VOC: La route des Indes hollandaises au XVII siècle (édition de Dink van der Gruysse), Chandeigne, coll. « Magellane », Paris, pp. 69-182 [ms. XVII ${ }^{\mathrm{e}}$ siècle]. 
KYrova Magda

1994 «Music in seventeenth-century Dutch painting » in Edwin Buijsen et LouisPeter Grijp (éd.), The Hoogsteder exhibition of music and painting in the Golden Age, Hoogsteder \& Hoogsteder, La Haye, pp. 3-61.

LABAT Jean-Baptiste

1993 Voyage aux isles: chronique aventureuse des Caraïbes 1693-1705, Phébus, coll. « Libretto », Paris [1698].

LEPPERT Richard D.

1975 Musical iconography and class attitude : 1600-1789, Musikverlag E. Katzbichler, Munich/ Salzbourg.

1977 Theme of music in Flemish paintings of the seventeenth century, 2 vol., Muzikverlag E. Katzbichler, Munich/Salzbourg.

1993 The sight of sound : music, representation, and the history of the body, University of California Press, Berkeley.

LESURE François

1954 "Les orchestres populaires à Paris vers la fin du $\mathrm{XVI}^{\mathrm{e}}$ siècle ", Revue de musicologie, 36, pp. 39-54.

Michel Wolfgang

1987 "Zacharias Wagner und Japan (I) - Ein Auszug aus dem "Journal des Donnermanns" ", Dokufutsu Bungaku Kenkyu, 37, pp. 53-101.

Mirimonde Albert Pomme de

1961 "Les sujets musicaux chez Vermeer de Delft", Gazette des Beaux-Arts, pp. 29-52.

1962 «La musique dans les œuvres hollandaises du Louvre. 1, Fêtes et scènes de genre », Revue du Louvre, pp. 123-138.

Moens Karel et Iris KochelBergh

1994 Muziek \& grafiek : burgemoraal en muziek in de 16de-en 17de-eeuwse Nederlanden : tentoonstelling, Antwerpen, Hessenhuis, 29 juli-30 oktober 1994, catalogue d'exposition, Pandora, Anvers.

MOREAu Pierre

1651 Histoire des derniers troubles du Brésil entre les Hollandois et les Portugais, Augustin Courbé, Paris.

MurPhy John Patrick

2008 Cavalo-marinho pernambucano, Editora Universidade Federal de Minas Gerais, Belo Horizonte.

Oliveira Carla Mary S.

2005a «Frans Post e as imagens do Brasil holandês: o olhar que registra e o traço que interpreta? », communication au Tercer congreso internacional Alexander Von Humboldt : literatura de viajes desde y hacia Latinoamerica del siglo XV al XXI, Centro universitario hispano-mexicano, Veracruz.

2005b «O Brasil seiscentista nas pinturas de Albert Eckhout e Frans Janszoon Post : documento ou invenção do Novo Mundo ? ", Actas do Congresso 
internacional atlântico de Antigo Regime : poderes e sociedades, Faculdade de ciências sociais e humanas, Universidade Nova de Lisboa, Lisbonne.

Oliveira Pinto Tiago de

2004 «Cem anos de etnomusicologia e a "era fonográfica" da disciplina no Brasil » in Anais do II Encontro Nacional da ABET (2004), São Paulo, pp. 103-124.

PyraRd DE Laval François

$1619 \quad$ Voyage de François Pyrard de Laval, contenant sa navigation aux Indes Orientales, Maldives, Moluques, Brésil : les divers accidens..., Samuel Thiboust et veuve Rémy Dallin, Paris [ $3^{\mathrm{e}}$ édition].

RiCHSHOFFER Ambrosius

2004 Diário de um soldado (1629-1632), Companhia Editora de Pernambuco, Recife [1677].

SANDRONI Carlos

2001 Feitiço decente. Transformações do samba no Rio de Janeiro (1917-1933), Jorge Zahar Editor/Editora UFRJ, Rio de Janeiro.

Schama Simon

1987 The embarassment of riches : an interpretation of Dutch culture in the Golden Age, Alfred A. Knopf, New York.

Sobrinho Paulo Fernandes RosA

2006 Sentidos e sonoridades múltiplas na música do coco do Recife e região metropolitana, mémoire de maîtrise d'anthropologie, Universidade Federal de Pernambuco, Recife.

SOUChU DE RENNEFORT Urbain

$1701 \quad$ Histoire des Indes orientales, Adrian Mœtjens, La Haye [1688].

TINHORÃo José Ramos

2000 As festas no Brasil colonial, Editora 34, São Paulo.

2008 Os sons dos Negros no Brasil. Cantos, danças, folguedos : origens, Editora 34, São Paulo [1988].

VIANNA Hermano

1999 O Mistério do Samba, Jorge Zahar Editor/Editora UFRJ, Rio de Janeiro [1995].

WÄJTEN Hermann

1938 O domínio colonial holandês no Brasil, Companhia Editora Nacional, São Paulo [1921].

ZuMThor Paul

1960 La vie quotidienne en Hollande au temps de Rembrandt, Hachette, Paris. 